\title{
SPARC-90: A Code for Calculating Fission Product Capture in Suppression Pools
}

Manuscript Completed: July 1991

Date Published: October 1991

Prepared by

P. C. Owczarski, K. W. Burk

Pacific Northwest Laboratory

Richland, WA 99352

\section{DISCLAIMER}

This report was prepared as an account of work sponsored by an agency of the United States Government. Neither the United States Government nor any agency thereof, nor any of their employees, makes any warranty, express or implied, or assumes any legal liability or responsibility for the accuracy, completeness, or usefulness of any information, apparatus, product, or process disclosed, or represents that its use would not infringe privately owned rights. Reference herein to any specific commercial product, process, or service by trade name, trademark, manufacturer, or otherwise does not necessarily constitute or imply its endorsement, recommendation, or favoring by the United States Government or any agency thereof. The views and opinions of authors expressed herein do not necessarily state or reflect those of the United States Government ot any agency thereof.

Prepared for

Division of Regulatory Applications

Office of Nuclear Regulatory Research

U.S. Nuclear Regulatory Commission

Washington, DC 20555

NRC FIN B2444

\section{MASTER}




\section{ABSTRACT}

This report describes the technical bases and use of two updated versions of a computer code initially developed to serve as a tool for calculating aerosol particle retention in boiling water reactor (BWR) pressure suppression pools during severe accidents, SPARC-87 and SPARC-90. The most recent version is SPARC-90. The initial or prototype version (Owczarski, Postma, and Schreck 1985) was improved to include the folloving: rigorous treatment of local particle deposition velocities on the surface of oblate spherical bubbles, new correlations for hydrodynamic behavior of bubble swarms, models for aerosol particle growth, both mechanistic and empirical models for vent exit region scrubbing, specific models for hydrodynamics of bubble breakup at various vent types, and models for capture of vapor iodine species.

A complete user's guide is provided for SPARC-90 (along with SPARC-87). A code description, code operating instructions, partial code 1 isting, examples of the use of SPARC-90, and summaries of experimental data comparison studies also support the use of SPARC-90. 


\section{EXECUTIVE SUMMARY}

This report describes the technical bases and use of two updated versions of a computer code initially developed to estimate particle retention in boiling water reactor (BWR) pressure suppression pools during severe accidents. The work was conducted by Pacific Northwest Laboratory (PNL) as part of a U.S. Nuclear Regulatory Commission (NRC) study of the fission product retention effectiveness of engineered safety features (ESFs).

The technical bases of the latest version of the code, SPARC-90, consist of the initial or prototype version (Owczarski, Postma, and Schreck 1985) with improveci algorithms, particle growth calculation methods, entrance effects for various orifice types, new heat and mass balances of bubbles rising in the pool, and new correlations for hydrodynamic behavior of bubble swarms.

This report is also intended to serve as user's manuals for SPARC-90 and SPARC-87. For this purpose, the following items are included: 1) code description with methods/algorithms of calculation and subroutines; 2) code operating instructions with input requirements, output descriptions, parameter selection guidelines, and examples of the code's use; 3 ) summaries of code versus experimental data comparison studies; and 4) a list of SPARC-90 input data subroutines. 


\section{ACKNOWLEDGMENTS}

The authors would like to take this opportunity to formally recognize the help received from the Electric Power Research Institute (EPRI) in providing information that aided in the preparation of this report.

Experimental data used for code comparisons was obtained from EPRI and ACE consortium sponsored pool scrubbing experiments managed by Battelle Columbus Laboratories $(\mathrm{BCL})$ and the Pacific Northwest Laboratory (PNL). The model data comparisons presented could not have been made without the information concerning test conditions that was graciously provided by EPRI, BCL, and PNL staff. 


\section{GLOSSARY OF TERMS}

a

A

$A_{1}-A_{4}$

ACE

AED

Al

ammd

b

$\mathrm{B}_{1}-\mathrm{B}_{6}$

$B C L$

BWR

C

$C_{S}^{\prime}$

C'́

$\mathrm{Cn}_{i}, \mathrm{Cn}_{j}, \mathrm{Cm}_{\mathrm{mi}}$

$\mathrm{C}_{\mathrm{pb}}$

$c_{V V}$

$\bar{C}_{v v}$

$C_{\mathrm{vnc}}$

$\bar{c}_{\text {vnc }}$

D

$\bar{d}_{\mathrm{a}}$ ellipse major axis or constant

bubble surface area or constant

experimental constants

advanced containment experiments

aerodynamic equivalent diameter

activity of the water solution

aerodynamic mass median diameter

ellipse minor axis or constant

parameters defined in terms of $a$ and $b$

Battelle Columbus Laboratories

boiling water reactor

parameter defined by $\left(a^{2}-b^{2}\right)^{1 / 2}$

heat capacity of solids

heat capacity of liquid water

Cunningham correction factor

heat capacity of the bubble at constant pressure

heat capacity of water vapor at constant volume

average values of heat capacity of water vapor over the range of temperature 273.2 to $T_{1}, K$

heat capacity of noncondensible gases at constant volume

average values of heat capacity of noncondensible gases over

the range of temperature 273.2 to $T_{1}, K$

vapor diffusivity in gas

area mean bubble diameter multiplier 
$d_{B}, d_{b} \quad$ equivalent sphere diameter, bubble equivalent volume sphere diameter

DF decontamination factor

DFec DF resulting from steam condensation

$\overline{\mathrm{DF}}_{i}, \overline{\mathrm{DF}}_{j} \quad$ cumulative $\mathrm{DF}$ for the entire process of each particle size $i, j$

DF I DF from impaction

DFov overall DF for all particle sizes

Dg bubble diameter

$\mathrm{Dg}_{i} \quad$ initial spherical globule diameter

$d_{j} \quad$ particle or bubble diameter of size $i$

$D_{i} \quad$ diffusion coefficient for particle size $i$ or inner pool diameter

$D_{j} \quad$ Brownian diffusion coefficient

$d_{j}, d_{0}^{\prime} \quad$ particle diameter

Do circulator vent diameter, outer pool diameter, opening diameter

$d_{v m} \quad$ volume mean diameter

E experimental parameter related to the formation frequency of a

bubble

$E_{C 1}, E_{C 2} \quad$ eccentricity

ESF engineered safety features

$f \quad$ friction factor

g gravitational acceleration constant or abbreviation for grams

mass

Gs swarm volume flow rate

$\mathrm{H} \quad$ partition coefficient

$h_{p} \quad$ vent depth 
van't Hoff ionization factor

Boltzmann's constant

gas thermal conductivity

m

mass of solute in the droplet

MD

mean difference

me

mass of condensed water as aerosol particles in bubble gas

MMD mass median diameter

Mnc

$\mathrm{m}_{\mathrm{S}}$

g-moles of noncondensible gas in bubble

Mv

$M_{t}$

$\mathrm{MW}$

MWo

MwV

N

$n_{j}$

$\mathrm{nL}$

NRC

$n_{T}$

$n_{W}$

$P$

$\dot{P}_{\operatorname{mi}}$

$\dot{P}_{\text {mo }}$

mass of solids as aerosol particles in bubble gas

g-moles of water vapor in the bubble

total moles of water vapor in the bubble $\left(M_{V}\right)+$ noncondensible

gas in bubble $\left(M_{n c}\right)$

molecular weight of solute

molecular weight of solvent

molecular weight of the vapor

total number of bubbles

number of bubbles of size $i$ in a flow cross-sectional volume, moles solute $i$ in droplet, number of particie of diameter $d_{i}$ number of molecules $/ \mathrm{cm}^{3}$ of solution

U.S. Nuclear Regulatory Commission

total moles

moles water in droplet

absolute gas pressure

particle mass flow rates of size $i$ particles into the pool

particle mass flow rates of size i particles out of the pool 


\begin{tabular}{|c|c|}
\hline PNL & Pacific Northwest Laboratory \\
\hline PT & pressure above the pool or total gas pressure in millibars \\
\hline Pv & actual vapor pressure of the water in the gas \\
\hline$P_{V}^{O}$ & $\begin{array}{l}\text { normal vapor pressure of water at the pressure and temperature } \\
\text { of the gas }\end{array}$ \\
\hline Pvb & vapor pressure of bubble \\
\hline Pvs & vapor pressure of water \\
\hline$P_{W}$ & pool vapor pressure \\
\hline$Q, \dot{Q}$ & gas volumetric flow rate or heat \\
\hline$r$ & radial coordinate or drop radius \\
\hline $\mathrm{R}$ & gas constant \\
\hline $\mathrm{R}^{2}$ & coefficient of correlation \\
\hline$r_{C}$ & surface radius of curvature \\
\hline s & length of arc \\
\hline$S$ & equilibrium saturation ratio (relative humidity) \\
\hline SE & standard error \\
\hline SPARC & Suppression Pool Aerosol Removal Code \\
\hline $\mathrm{S}_{r}$ & saturation ratio at particle surface \\
\hline Stki & Stokes number \\
\hline$t$ & time \\
\hline T & absolute temperature \\
\hline$T_{b}$ & bubble temperature \\
\hline$t_{b}$ & bubble rise time or residence time of bubbles in the pool \\
\hline$t_{e}$ & exposure time of the moving surface \\
\hline$t_{f}$ & globule filling time \\
\hline$T$ & pool temperature \\
\hline
\end{tabular}




\begin{tabular}{|c|c|}
\hline U & internal energy \\
\hline v & droplet radius \\
\hline$\tilde{v}$ & liquid molar volume \\
\hline$v_{b}$ & globule velocity \\
\hline$V_{B}, V_{D}$ & Brownian diffusion velocity \\
\hline Vbo & detachment velocity \\
\hline$v_{c}$ & centrifugal deposition velocity \\
\hline$v_{e}$ & vent exit gas velocity \\
\hline$v_{g}$ & gravitational settling velocity \\
\hline$v_{n}$ & net local deposition velocity \\
\hline$v_{0}$ & exit velocity of the gas \\
\hline$v_{r}$ & relative velocity of gas to liquid/bubble rise velocity \\
\hline $\bar{v}_{\mathbf{S}}$ & average bubble swarm velocity \\
\hline$v_{s}$ & settling velocity \\
\hline$v_{\text {SW }}$ & swarm rise velocity \\
\hline$v_{v}$ & vapor velocity \\
\hline We & Weber number \\
\hline$x$ & vertical distance above vent exit \\
\hline$x_{b}$ & water vapor mole fraction in bubble \\
\hline$x_{i}$ & mole fraction of noncondensible gas in inlet gas \\
\hline$x_{n c}$ & mole fraction of noncondensible gas in the gas entering the pool \\
\hline$x_{0}$ & $\begin{array}{l}\text { mole fraction of noncondensible gas in the gas after it attains } \\
\text { thermal and vapor equilibrium in the pool at the inlet depth } \\
\text { water vapor mole fraction at interface }\end{array}$ \\
\hline
\end{tabular}




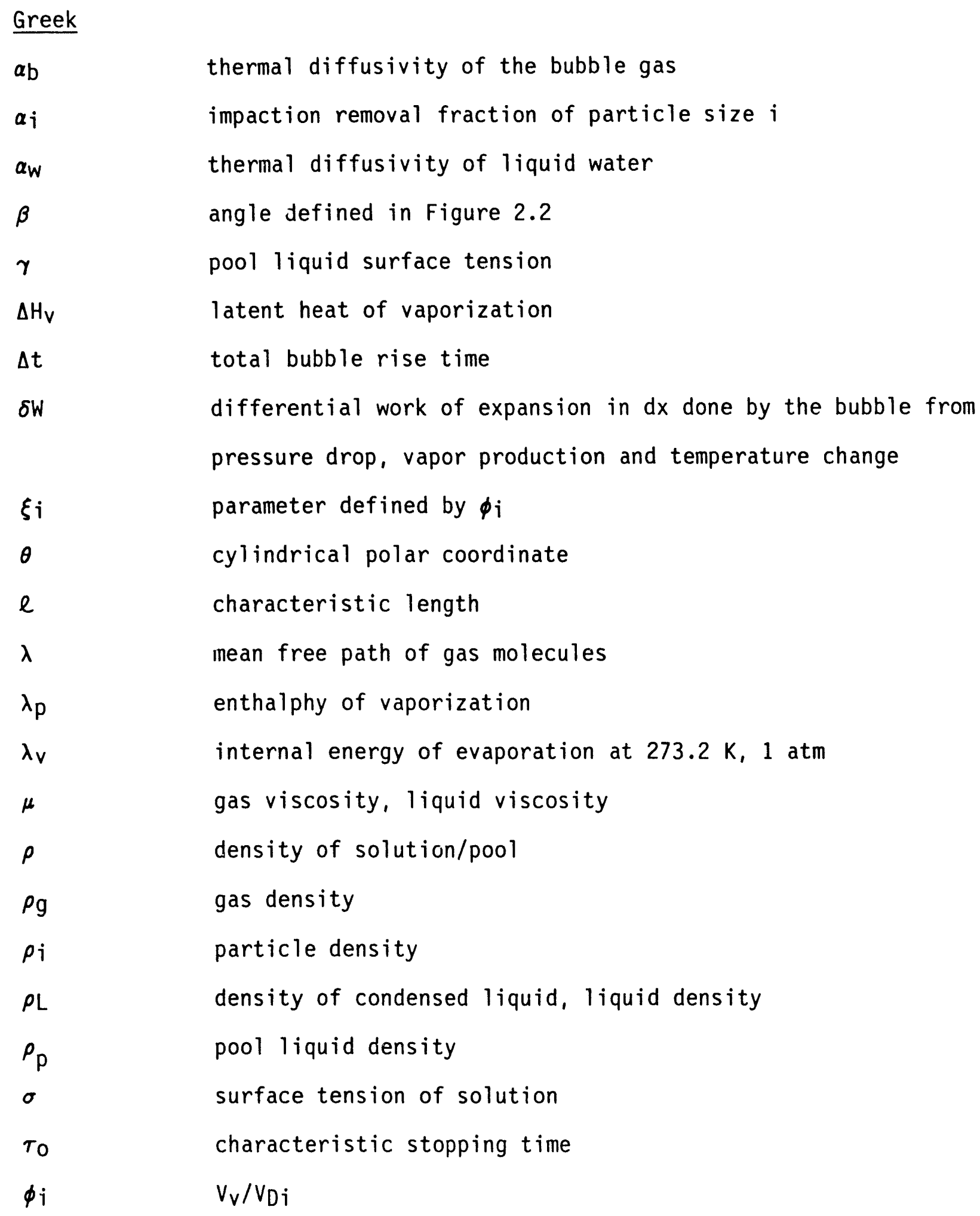

$a_{b}$

$\alpha_{i}$

$a_{w}$

$\beta$

$\boldsymbol{\gamma}$

$\Delta H_{V}$

$\Delta t$

$\delta W$

$\xi i$

$\theta$

e

$\lambda$

$\lambda_{p}$

$\lambda_{v}$

$\mu$

$\rho$

$\rho_{g}$

$\rho i$

$\rho L$

$\rho_{p}$

$\sigma$

To

$\phi_{i}$

thermal diffusivity of the bubble gas

impaction removal fraction of particle size $i$

thermal diffusivity of liquid water

angle defined in Figure 2.2

pool liquid surface tension

latent heat of vaporization

total bubble rise time

differential work of expansion in $\mathrm{dx}$ done by the bubble from

pressure drop, vapor production and temperature change

parameter defined by $\phi_{i}$

cylindrical polar coordinate

characteristic length

mean free path of gas molecules

enthalphy of vaporization

internal energy of evaporation at $273.2 \mathrm{~K}, 1 \mathrm{~atm}$

gas viscosity, liquid viscosity

density of solution/pool

gas density

particle density

density of condensed liquid, liquid density

pool liquid density

surface tension of solution

characteristic stopping time

$V_{V} / V_{D i}$ 
normalized globule volume 


\section{CONTENTS}

ABSTRACT .............................. $i$ i

EXECUTIVE SUMMARY ................... . . .

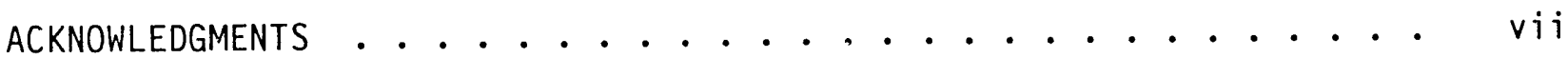

GLOSSARY OF TERMS ........................ ix

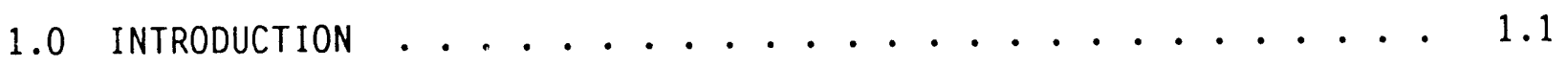

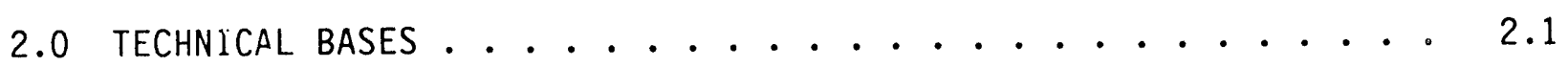

2.1 DESCRIPTION OF POOL PROCESSES . . . . . . . . 2.1

2.1 .1 Hydrodynamics ................. 2.1

2.1 .2 Thermal Hydraulics ............ 2.2

2.1 .3 Aerosol Capture ............. . . 2.3

2.1 .4 Volatile Iodine Species Capture ....... 2.5

2.1 .5 Entrainment . . . . . . . . . . 2.7

2.2 MODELS FOR POOL PROCESSES . . . . . . . . . . . . 2.8

2.2 .1 Hydrodynamics . . . . . . . . . 2.8

2.2.2 Aerosol Capture ............. . . 2.12

2.2.3 Thermal Hydrautics ............ 2.19

2.2.4 Particle Growth, SPARC-87 . . . . . . . 2.24

2.2.5 Particle Growth, SPARC-90 . . . . . . . 2.28

3.0 SPARC-90 DESCRIPTION . . . . . . . . . . . . . . 3.1

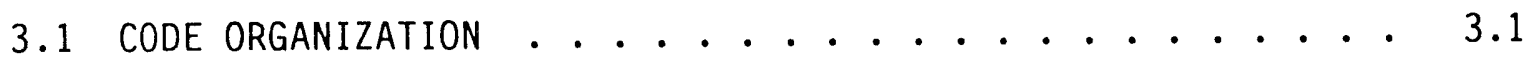

3.1 .1 Read Input File................. 3.1

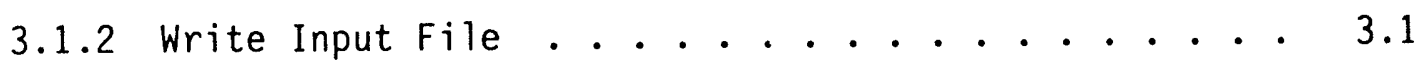

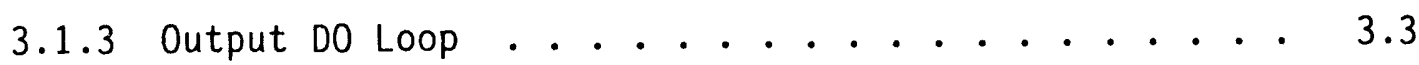

3.1 .4 Input Adjustment .................. 3.3

3.1.5 DF Entrance Region .......... 3.3

xvi i 
3.1.6 Swarm Rise DO Loop ............. . 3.3

3.1.7 Thermodynamic Conditions ........... . 3.3

3.1.8 Particle-Growth Calculations . . . . . . . 3.3

3.1.9 Bubble Rise DF Calculations . . . . . . . . . 3.4

3.1.10 Cumulative DF ................. 3.4

3.1.11 Output Parameter Calculations . . . . . . . . 3.5

3.2 Calculational Subroutines . . . . . . . . . . . . . . 3.5

3.2.1 Subroutine DIFFU . . . . . . . . . . . 3.5

3.2.2 Subroutine KELVIN . . . . . . . . . . . 3.5

3.2.3 Subroutine I2EQUIL . . . . . . . . . . 3.5

3.2.4 Subroutine SOLGRO . . . . . . . . . . . . 3.6

3.2 .5 Subroutine REQUIL . . . . . . . . . . . . 3.6

3.2.6 Subroutine PSI2ABS . . . . . . . . . . 3.6

3.2.7 Subroutine PGROW . . . . . . . . . . . . 3.6

3.2.8 Subroutine DFVENT . . . . . . . . . . . . 3.6

4.0 CODE OPERATION AND PARAMETER SELECTION . . . . . . . . . . . . 4.1

4.1 INPUT REQUIREMENTS . . . . . . . . . . . . . . . . 4.1

4.1.1 Pool Physical Parameters ........... . 4.1

4.1.2 Inlet Aerosol Size Distribution Parameters . . . . 4.1

4.1.3 Vent Paraneters ............... 4.2

4.1.4 Aerosol Species and Other Chemical Species

4.1.5 Calculational and Outout Specifications...... 4.3

4.1.6 Order of Input Values . . . . . . . . . . . 4.3

4.2 OUTPUT DESCRIPTION . . . . . . . . . . . . . . . . . 4.4

4.2.1 Output Description by Particle Size . . . . . . 4.5 
4.2.2 Additional Output ................ 4.5

4.3 INPUT/OUTPUT EXAMPLES - SAMPLE PROBLEM . . . . . . . 4.5

4.4 PARAMETER SENSITIVITY STUDY . . . . . . . . . . . 4.5

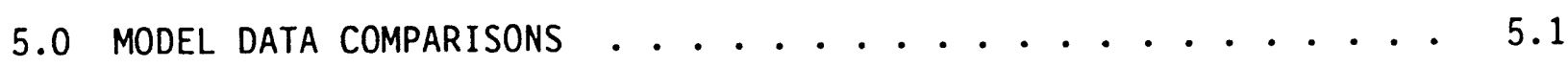

5.1 INITIAL COMPARISON ....................... 5.1

5.1 .1 Initial Data Base ........... 5.1

5.1 .2 Comparison Criteria .............. 5.1

5.1 .3 SPARC-87 Models .............. 5.2

5.1 .4 Initial Comparison Results ........ 5.2

5.1.5 SPARC Iodine Scrubbing Comparisons ....... 5.3

5.2 SPARC-87 COMPARISON .......................... 5.4

5.3 SPARC-90 COMPARISON ................... 5.6

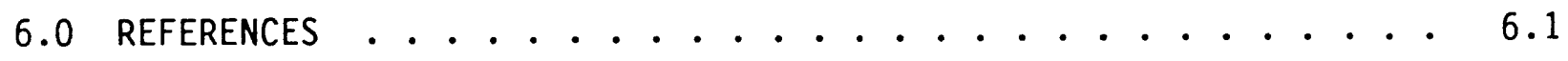

APPENDIX - SPARC-90 LISTING OF INPUT SUBROUTINES . . . . . . A.1 


\section{FIGURES}

2.1 Schematic of Suppression Pool During Scrubbing of Inlet Gases........................ 2.1

2.2 Rising Oblate Spheroid Bubble Showing the Constant Vapor Concentration Isopleth, Various Velocities, and Ellipse

Parameters . . . . . . . . . . . . . . . 2.4

3.1 Flow Chart of the SPARC-90 Code . . . . . . . . . . . 3.2

$5.1 \mathrm{Log} / \log$ Plot of the Two DF Data Sets of Tables 5.1 and 5.2 . . 5.5 


\section{$\underline{\text { TABLES }}$}

2.1 Summary of Entrainment Calculations . . . . . . . . . . 2.8

2.2 Growth of $\mathrm{CsOH}$ Particles in Humid Atmosphere at $100^{\circ} \mathrm{C}$ with I=2.................... 2.25

4.1 Example of an Input file . . . . . . . . . . . . . . 4.6

4.2 SPARC Printoui of Table 4.1 Input file . . . . . . . . . . 4.7

4.3 Particle-Size Output Data for Table 4.1 Input File at 0.0 min. $\quad 4.8$

4.4 Overall Aerosol Properties and Other Parameters - Part of the Output File for Table 4.1 Input File at $0.0 \mathrm{~min} .$. . . . . 4.9

4.5 Parameter Sensitivity Summary . . . . . . . . . . . . 4.10

5.1 SPARC-87/ACE DF Comparisons . . . . . . . . . . . . . 5.4

5.2 SPARC-87/BCL DF Comparisons . . . . . . . . . . . . 5.5

5.3 SPARC-90/ACE DF Comparisons . . . . . . . . . . . . 5.6

5.4 SPARC-90/BCL DF Comparisons ............... . 5.7 


\subsection{INTRODUCTION}

The U.S. Nuclear Regulatory Commission (NRC) is studying the fission product retention effectiveness of engineered safety features (ESFs) in light water reactors under postulated severe accident conditions. These conditions could result from a highly degraded core and possible interactions between the core and the concrete basemat. Elevated temperatures, high pressures, and very high aerosol particle concentrations (up to $10 \mathrm{~g} / \mathrm{m}^{3}$ ) might be characteristic of reactor containment atmospheres. The responses of various ESF systems to the severe conditions are being examined both by modeling and by experiments. This report describes the analytical models developed to estimate the extent of retention in boiling water reactor (BWR) pressure suppression pools.

Pacific Northwest Laboratory (PNL) produced a prototypic Suppression Pool Aerosol Removal Code (SPARC), described in NUREG/CR-3317 (OwCzarsk i, Postma, and Schreck 1985) that contains several models for aerosol particle depletion and thermal hydraulics. This computer code was developed to calculate the capture of aerosol particles by the wet well (pressure suppression pool) of a BWR during hypothetical accidents in which aerosol from a degraded core or core/concrete reaction is forced through the pool.

In the years following the development of the prototypic version of SPARC, several improved calculational methods (algorithms) were used to modify the code. These include 2 more rigorous treatment of all particle deposition mechanisms and the hindering vapor influx during bubble rise. Also, new data were made available that helped produce improved models for the hydrodynamic description of bubble swarms. A notable improvement, however, results from the recognition that the expansion work of rising bubbles in a pool produces cooler-than-pool bubbles that have supersaturated atmospheres. This supersaturation can lead to significant particle growth, which can enhance pool decontamination by several orders of magnitude under certain conditions. Also added are vent region scrubbing models, bubble breakup models for each vent type, and detailed particle growth models. Two versions of SPARC (87 and 90) are discussed. The 87 version is more empirical, whereas the 90 version is more mechanistic in particle growth and vent exit scrubbing. Because these versions are identical except in these two areas, information presented in the report will be related to SPARC-90 unless noted otherwise.

The objectives of this document are to

- discuss the technical bases of the two latest version of SPARC

- describe the code

- provide instructions for its use.

Section 2 first qualitatively describes the pool processes, then summarizes the mathematical details of the process models. These models include the pertinent pool hydrodynamics, aerosol capture, bubble thermodynamics and heat 
and mass transfer, particle growth, and pool entrainment. Section 3 describes the code and its subroutines. Section 4 provides the details for using the code including input requirements, output description, parameter selection guidelines and examples of SPARC-90 use. Section 5 contains comparisons of code calculations with experimental results (model data comparisons). The appendix contains a listing of pertinent SPARC-90 subroutines for creating input data files.

Copies of the code will be made available to interested users upon request to the author. It is also planned to make the code available through the Argonne Code Center. Although some model-data comparisons have been made that suggest reasonable agreement between experimental and code-generated data, the code cannot be described as fully validated. No assurance is expressed or inplied as to the accuracy or completeness of the results. 


\subsection{TECHNICAL BASES}

This section discusses the essential technical models of SPARC-90. A brief qualitative discussion of the scrubbing process in suppression pools is followed by detailed model descriptions of the pool processes pertinent to particle capture.

\subsection{DESCRIPTION OF POOL PROCESSES}

This section gives a qualitative discussion of the action of the gases passing through the suppression pool from a submerged vent to the pool surface. Hydrodynamic and thermal-hydraulic processes and retention mechanisms associated with particle capture are discussed along with aspects of volatile iodine behavior related to pool scrubbing.

\subsubsection{Hydrodynamics}

Several aspects of the hydrodynamic processes are important to the scrubbing process. Figure 2.1 is a schematic of the suppression pool during the scrubbing of gases. The inlet gases can enter the pool through a variety of vents in the various BWR systems. During steady flow, the gases leaving the vent form large globules that break up into a swarm of small bubbles within a few globule diameters from the vent (see last paragraph this section). This swarm rises to the pool surface in several seconds depending on inlet noncondensible gas flow rate and pool depth. The individual bubbles frequently coalesce and break up during the bubble rise period. This bubble interaction keeps the bubbie size distribution constant, even though the volume of gas increases in the swarm as it rises to the surface (Paul et al. 1985).

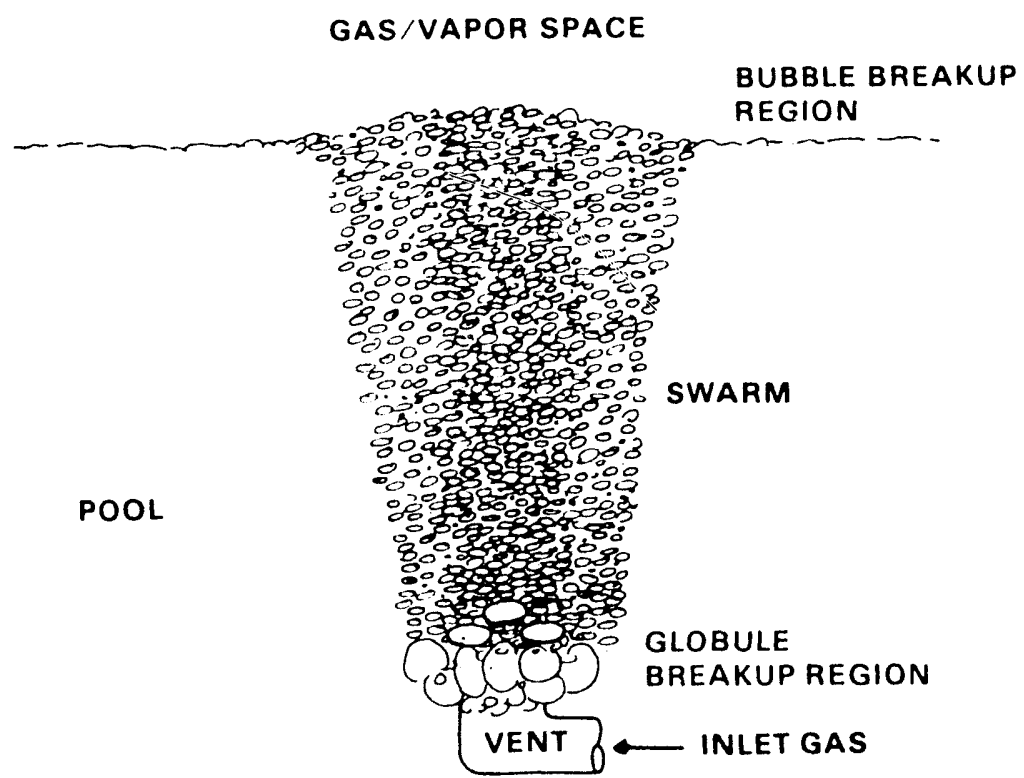

FIGURE 2.1. Schematic of Suppression Pool During Scrubbing of Inlet Gases 
The average swarm velocity and the swarm diameter also increase as the swarm rises to the surface. The swarm column serves as a pool 'pump' that recirculates the pool water and helps keep it well-mixed.

Individual bubbles in the swarm are oblate spheroids whose flatness depends on bubble size. Each bubble wobbles from side to side as it rises. The viscous shear of the liquid in relative motion past the bubble causes the bubble surface and interior to move in a top-to-bottom rotation. This movement or circulation is the main mechanical action that scrubs large particles from the bubble via centrifugal force. As the bubbles reach the surface, most of them break up (some might recirculate with the pool fluid). The breakup process produces droplets; some are small enough to be carried away (entrained) by the pool gases. The quantitative role of entrainment is addressed in Section 2.1.5 and the quantitative treatment of bubble size and shape is discussed in Section 2.2.1.

The size of the globule initially formed at the vent depends on the vent type (e.g., horizontal vent, downcomer, or T-quencher) and the noncondensible gas flow rate. The unstable globule then breaks up as it rises. Breakup begins almost immediately for small orifices. For large vents, tests have revealed that globules must travel a vertical distance of about 10 to 12 times their initial diameter before breakup is complete.

\subsubsection{Thermal Hydraulics}

The thermal hydraulics of the pool/gas interaction also affects particle capture. Three different aspects of thermal hydraulics are involved: heat transfer to/from the gas phase, mass transfer to/from gas phase, and the work of the expanding gas phase as the bubble rises.

The gas entering the pool through the vents rapidly equilibrates to the pool temperature as it breaks up into bubbles. Moody and Nagy (1983) have estimated this equilibration time to be typically about $0.03 \mathrm{~s}$. In SPARC-90, equilibration is assumed to be instantaneous for both temperature and vapor pressure. A large temperature gradient between gas and pool can cause thermophoretic movement of particles. This effect is currently ignored because the gradient is usually short-lived and of small magnitude. However, net transfer of vapor mass across the gas/liquid boundary during equilibrium either can carry particles to the boundary (if condensation occurs at the boundary) or hinder particle movement to the boundary (if evaporation occurs). This mechanism is called Stefan flow and is usually considered along with diffusiophoresis, the particle transfer mechanism involving molecular weight gradients. Hot dry gases entering the pool evaporate water and hinder particle capture, whereas high steam fractions in the inlet gas would enhance particle capture. These entrance effects (Owczarski, Postma, and Schreck 1985) are discussed quantitatively in Section 2.2.

As the bubble swarm rises, vapor transfers into the bubble to try to maintain vapor equilibrium (Owczarski, Postma, and Schreck 1985). This 
entering vapor not only hinders particle capture by the pool, but also adds to the swarm volume and provides more steam for particle growth by condensation.

Condensation of water vapor on particles occurs if the vapor pressure of the bubble is greater than the vapor pressure of wet particles. Dissolved particles have lower vapor pressure than wet insoluble particles. Therefore, the potential for growth of soluble particles exists even in subsaturated atmospheres. Algorithms in SPARC -90 account for this type of soluble particle growth (Section 2.2.5).

Supersaturated atmospheres in the bubbles exist throughout the bubble rise period. This supersaturation is caused by the cooling of the bubbles as they do expansion work while rising to the surface. If the bubbles were adiabatic, the cooling potential would result in about a $10^{\circ} \mathrm{C}$ temperature drop for an air bubble rising in a $100^{\circ} \mathrm{C}$ pool from a depth of $1 \mathrm{~m}$ to the surface where the absolute pressure is $1 \mathrm{~atm}$. Because the bubbles are not adiabatic (both sensible heat and latent heat transfers into the bubble), the actual temperature drop would be less than $10^{\circ} \mathrm{C}$. The details of the model that SPARC90 uses to simulate condensation on particles in supersaturated atmospheres are given in Section 2.2.3.

\subsubsection{Aerosol Capture}

Particle capture preceding swarm formation is caused by the following mechanisms:

1. Excess steam (above the pool vapor pressure) condensation moves particles to the surface.

2. Vent exit gas temperatures higher than pool temperatures cause thermophoretic deposition.

3. High vent exit velocities cause inertial impaction on pool water.

4. Bubble formation, involves curved surface motion and potential particle capture.

Thermophoresis is currently ignored in SPARC-90, and inlet velocities need to be near sonic velocities to affect inertial particle capture.

Removal of particles during the bubble rise period is caused by inertial deposition, sedimentation, and diffusion. The shape that best represents the stable bubbles during this rise period is an oblate spheroid. The larger bubbles are flatter than the smaller bubbles. For a given bubble size the relative velocity of gas to liquid $\left(V_{r}\right)$ determines this shape. Figure 2.2 shows a typical vertical cross-section of a swarm bubble through its center--an ellipse. Isolated bubbles (not in a swarm) can be flattened into lenticular 
bubbles. In pure water, bubbles exhibit free surface circulation (at velocity $V_{s}$ ) caused by the relative fluid velocities. This circulation greatly aids the capture of particles by centrifugal forces and by the destruction of the diffusion boundary layers of the outwardly diffusing (Brownian) particles and the inwardly diffusing water vapor. The net local deposition velocity $\left(V_{n}\right)$ from all forces is the vector sum of the centrifugal deposition velocity $\left(V_{C}\right)$, gravitational settling velocity $\left(V_{g}\right)$, incoming vapor velocity $\left(V_{V}\right)$, and Brownian diffusion velocity $\left(V_{B}\right)$ :

$$
v_{n}=v_{c}+v_{v}+v_{g} \cos \beta+v_{B}
$$

This net deposition velocity can be used to define the decontamination factor (DF) for the three particle capture mechanisms. The decontamination factor for a process is the mass flow rate of particles into the process divided by the mass flow rate of particles out of the process. The velocity $\left(V_{n}\right)$ is

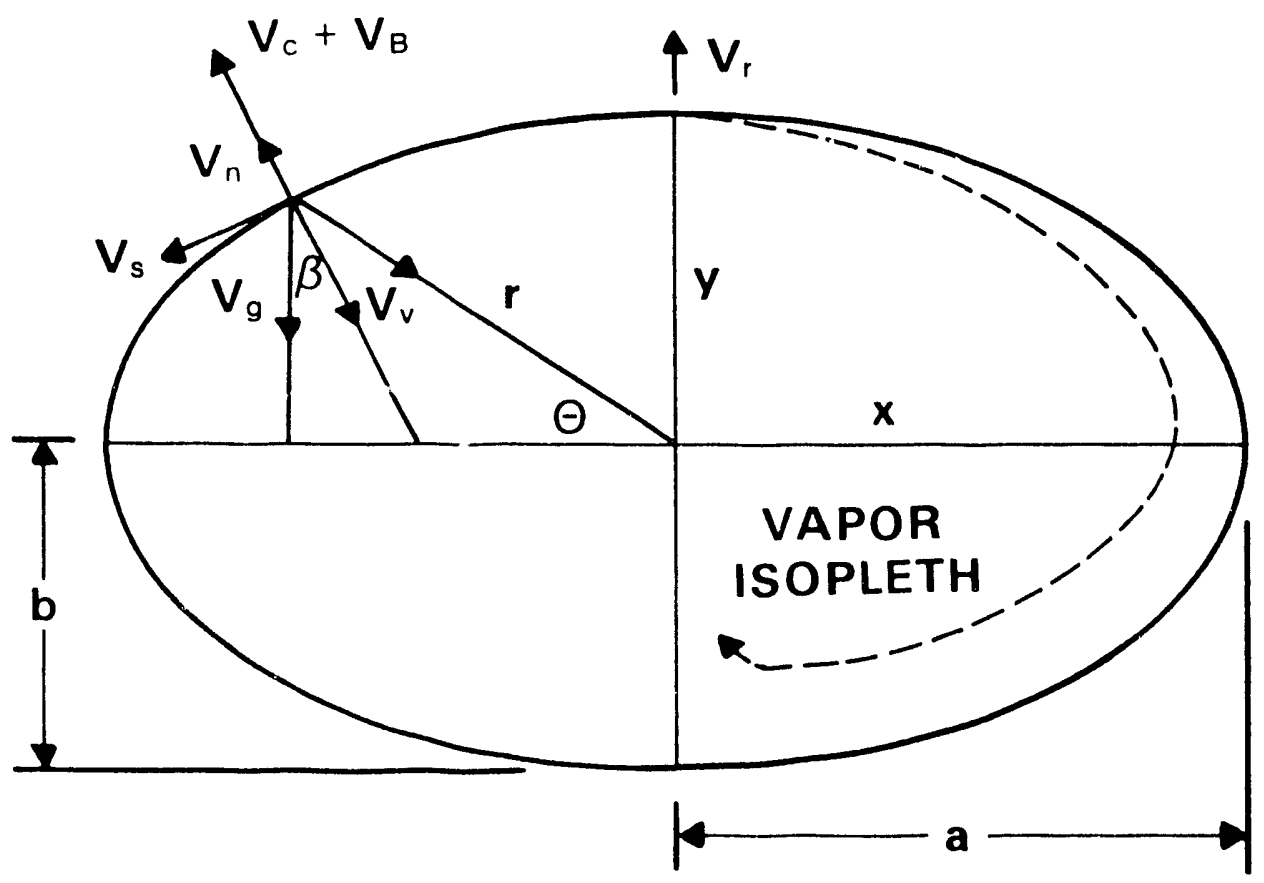

FIGURE 2.2. Rising Oblate Spheroid Bubble Showing the Constant Vapor Concentration Isopleth, Various Velocities, and Ellipse Parameters 
integrated over the whole bubble surface area and over the bubble rise time $\left(t_{b}\right)$,

$$
D F=\exp \left[\int_{0}^{t_{b}} \int_{A} v_{n} d A d t / \pi a^{2} b\right]
$$

At the pool surface, if the bubbles have any lifetime, gravity settling and Brownian diffusion can remove particles. Particle retention at the pool surface is currently not considered in SPARC-90 because of the lack of suitable data on the lifetime of surface bubbles.

To obtain the overall DF for a single particle size during the entire pool/gas contact period, the above DF is multiplied by the DF during initial globule formation. The models and the equations to describe and simulate these pool processes are discussed in Section 2.2.

\subsubsection{Volatile Iodine Species Capture}

The effectiveness of suppression pools in capturing iodine species was studied with SPARC (Owczarski and Winegardner 1986). The general conclusions were that I2 and HI would be captured with high DFs, and organic iodides would be captured lightly until the pool was saturated. The various aspects of iodine behavior are discussed below.

A number of aspects of iodine behavior are related to its capture in suppression pools. These aspects can be identified in three gas flow regions. The first region is the flow of iodine species in the core-melt off gases in the reactor primary system. The second is the vent exit region in the pool and the third is the bubble swarm rise region in the pool.

In the primary system, where gases are hydrogen and steam and iodine species can be I2, organic iodides, HI, and particulate iodides such as CsI, conditions can exist that favor the complete removal of the volatile inorganic species from the gas phase. These favorable conditions occur where temperatures are sufficiently low to allow alkaline aerosol particles to exist as a liquid or partially liquid phase. Alkaline hydroxides such as $\mathrm{CsOH}$ have this property in the vicinity of $300^{\circ} \mathrm{C}$ (Rollet, Cohen-Adad, and Ferlin 1963). This liquid phase can be highly reactive with the volatile species $\mathrm{HI}$ and $\mathrm{I}_{2}$. It is theorized that solid $\mathrm{CsOH}$ can be reactive with these species as well. The SPARC code has a subroutine that allows the user to switch on this iodine absorption process in the primary system. The process is modeled as a continuous plug-flow reactor where spherical aerosol particles absorb elemental iodine at a rate controlled by the diffusion of $I_{2}$ in the gas phase around the particles. Although not modeled, HI would behave similarly to I2, but with a slightly higher diffusion coefficient. 
As the gases leave the primary system, they enter the pool at depth through a specific vent type. In the region of this vent, the gases tend to equilibrate with the thermodynamic conditions of the pooi at the vent depth. This equilibration process frequently results in steam condensation and scrubbing of particles. In SPARC, this condensation results in some deposition of $\mathrm{I}_{2}$ and $\mathrm{CH}_{3} \mathrm{I}$, but the process is limited by the species solubility at the interface.

After the initial gas globules at the vent break up into the rising bubble swarm, the SPARC code assumes that bubble circulation continually renews the bubble interface and that the film theory of mass transfer resistance holds on botr sides of the interface. The equilibrium boundary conditions at the interface for the two volatile iodine species are (brackets refer to concentrations):

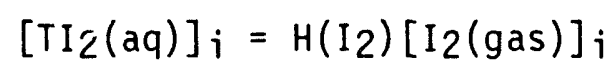

and

$$
\left[\mathrm{CH}_{3} \mathrm{I}(\mathrm{aq})\right]_{i}=\mathrm{H}\left(\mathrm{CH}_{3} \mathrm{I}\right)\left[\mathrm{CH}_{3} \mathrm{I}(\text { gas })\right]_{i}
$$

where $\left[\mathrm{TI}_{2}(\mathrm{aq})\right]_{i}=$ total liquid molar concentration of iodine at the interface as $\mathrm{I}_{2}$

$\left[I_{2} \text { (gas) }\right]_{i}=$ interfacial gas molar concentration of $I_{2}$

$H\left(I_{2}\right)=$ iodine partition coefficient.

Similar definitions hold for $\mathrm{CH}_{3} \mathrm{I}$.

The aqueous chemistry of iodine is controlled by the fast reactions (Eggleton 1967)

$$
\begin{aligned}
& \mathrm{I}_{2}(\mathrm{gas})=\mathrm{I}_{2}(a q) \\
& \mathrm{I}_{2}(a q)+\mathrm{I}^{-}=\mathrm{I}_{3}^{-} \\
& \mathrm{I}_{2}(\mathrm{aq})+\mathrm{H}_{2} \mathrm{O}=\mathrm{H}^{+}+\mathrm{I}^{-}+\mathrm{HIO}^{-} \\
& \mathrm{I}_{2}(\mathrm{aq})+\mathrm{H}_{2} \mathrm{O}=\mathrm{H}_{2} \mathrm{OI}^{+}+\mathrm{I}^{-} \\
& \mathrm{H}_{2} \mathrm{O}=\mathrm{H}^{+}+\mathrm{OH}^{-}
\end{aligned}
$$


By using the equilibrium constants for the above five reactions, the partition coefficient is quantitatively defined if mass balances of all iodine species and $\mathrm{H}^{+}$and $\mathrm{OH}^{-}$are maintained. The value of $\mathrm{H}\left(\mathrm{CH}_{3} \mathrm{I}\right)$ is obtained in a simpler way using solubility and vapor pressure data (Glew and Moelwyn-Hughes 1953).

The above concentrations are affected in time by certain slow aqueous reactions. Of particular interest is the lowering of the $\mathrm{pH}$ of aqueous solutions by radiation fields. The radiation-induced $\mathrm{pH}$ changes are not modeled in SPARC where they would effectively lower the H(I2) partition coefficient making $I_{2}$ and $H I$ more difficult to scrub. However, if accident sequences provide excess $\mathrm{CsOH}$ as expected (remains at high $\mathrm{pH}$ ), the SPARC models might still be adequate.

\subsubsection{Entrainment}

Aerosol droplets are produced when swarm bubbles break up at the pool surface forming new aerosol particles. If the entrainment particle production rate multiplied by duration times pool concentration produces significant quantities of aerosol radionuclides from materials previously trapped in the pool, an entrainment model should be included. However, the scoping calculations, discussed below, dismissed the need for such a model.

The correlations used for the scoping calculations come from Kataoka and Ishii (1983). This document provides correlations for both entrainment rates and particle size distributions for the entrained droplets. These correlations require the gas volume flow rate as well as liquid and gas physical properties.

Mark I BWR suppression pool parameters were used in the calculations. MARCH code runs (Gieske et al. 1984) and SPARC-90 runs were used to obtain flow rates (Table 2.1). From the MARCH runs, the maximum flow rate into the pool occurs during core melt with the metal/water reactions producing $\mathrm{H}_{2}$ and steam. The $\mathrm{H}_{2}$ gas flow ranges from 0 to $500 \mathrm{lb} / \mathrm{min}$. This flow rate is divided into 96 vents, each at the $4-\mathrm{ft}$ depth. The total mass of $\mathrm{H}_{2}$ that can be formed is $6000 \mathrm{lb}$. In SPARC-90 runs, the hydrogen gas leaving the pool is slightly supersaturated with approximately $90 \%$ water vapor by volume. This provides a gas volume rate of $6.0 \mathrm{~m} / \mathrm{s} /$ vent breaking the surface above each vent for the maximum $\mathrm{H}_{2}$ flow of $500 \mathrm{lh} / \mathrm{min}$ (with $4050 \mathrm{lb} \mathrm{H} 2 \mathrm{O}$ vapor/min), which could last only 12 min. Entrainment rates, total entrained mass, and maximum particle size are tabulated below for two cases: maximum flow (12 min) and 0.1 maximum flow $(120 \mathrm{~min})$.

The fraction of the pool entrained (ii.65 E-04) in this maximum flow case is insignificant for many reasons:

1. The maximum flow rate will not occur as a square wave. Much of the $\mathrm{H}_{2}$ flow will be at lower rates (and far lower entrainment rates). The entrainment rate is approximately proportional to (volumetric flow rate) 2 . 
TABLE 2.1. Summary of Entrainment Calculations

\begin{tabular}{|c|c|c|c|c|c|c|}
\hline Case & $\begin{array}{c}\text { Flow Rate } \\
\text { Per Vent, } \\
\mathrm{m}^{3} / \mathrm{s} \\
\end{array}$ & $\begin{array}{l}\text { Dura- } \\
\text { tion, } \\
\text { min } \\
\end{array}$ & $\begin{array}{c}\text { Entrain- } \\
\text { ment Rate, } \\
\mathrm{g} / \mathrm{s} \\
\end{array}$ & $\begin{array}{c}\text { Total } \\
\text { Entrained, } \\
\mathrm{g} \\
\end{array}$ & $\begin{array}{l}\text { Fraction } \\
\text { of Pool }(a) \\
\end{array}$ & $\begin{array}{l}\text { Maximum } \\
\text { Particle } \\
\text { Size, } \mu \mathrm{m} \\
\end{array}$ \\
\hline $\begin{array}{l}\text { 1. Maximum } \\
\text { flow rate }\end{array}$ & 6 & 12 & 2050 & 24,600 & $6.65 E-04$ & 200 \\
\hline $\begin{array}{l}\text { 2. } 0.1 \text { Max- } \\
\text { imum flow } \\
\text { rate }\end{array}$ & 0.6 & 120 & 1.55 & 186 & $5.05 E-06$ & 50 \\
\hline
\end{tabular}

(a) Pool liquid is $3.7 \times 10^{7} \mathrm{~g} \mathrm{H}_{2} \mathrm{O}$.

2. All of the important fission product mass will not be in the pool before $\mathrm{H}_{2}$ flow commences. As a general rule, the fission product aerosols are generated simultaneously with the $\mathrm{H}_{2}$ gas.

3. The droplets generated at the maximum flow rate are very large and the mass distribution function is at its peak at the largest particle [200 $\mu \mathrm{m}$ aerodynamic mass median diameter (amm)]. If no droplet evaporation occurs, particles $>20 \mu \mathrm{m}$ ammd will rapidly fall out when the $\mathrm{H}_{2}$ flow slows down or when the aerosol above the pool flows to another compartment.

4. Although the core/concrete interaction generates additional noncondensible gases, the flow rates are generally much less than the $\mathrm{H}_{2}$ flow rate.

5. Calculations indicate that pool scrubbing DF values will frequentiy be much less than $1500(1 / 6.65 \mathrm{E}-04)$. When the pool DF is less than 1500 , the penetration rate of unscrubbed radionuclides through the pool will certainly exceed any pool generated entrainment rate of radionuclides.

For the above reasons, no entrainment model has been programmed into SPARC-90.

\subsection{MODELS FOR POOL PROCESSES}

This section begins with the quantitative representations of the hydrodynamic processes. Aerosol capture models follow and, lastly, the models for thermal hydraulics and particle growth are described.

\subsubsection{Hydrodynamics}

The SPARC-90 version describes the pertinent hydrodynamic relationships with empirical equations that use the essential controlling parameters as independent variables. The hydrodynamic parameters needed are the initial bubble diameter and stable bubble size, shape, and rise velocity relative to rising fluid, and swarm rise velocity relative to the pool. 


\section{Initial Globule Diameter}

The Electric Power Research Institute hydrodynamic tests conducted at Battelle Columbus Laboratories produced valuable vent exit data that have been incorporated into SPARC as correlations. Each vent type studied (multihole vents, e.g.., "T" and "X" quenchers; downcomers; and horizontal vents) had unique sizes of initial globules formed at the vent. These globules rose and broke up into the smaller bubbles of the stable bubble swarm.

The first pool process correlation used in SPARC is based on the observation that the initial globule of volume $v_{g}$ broke up completely into the stable bubbles after rising 10 to 12 globule diameters (Paul et al. 1985) regardless of the initial size or vent type. This is represented in SPARC as a linear decrease in bubble diameter $\mathrm{Dg}$ in 12 initial spherical diameters, $\mathrm{Dg} j$.

$$
D g=D g_{i}\left(1.0-x / 12 D g_{j}\right)
$$

where $x$ is the vertical distance above the exit. Because $\nu_{g}=\pi \mathrm{Dg}^{3} / 6$, the fraction of gas in the unstable globule rapidly decreases in distance above the vent. A significant assumption (for horizontal vents and downcomers only) is made about this unstable globule, i.e., it is assumed that the scrubbing processes in this globule are insignificant (DFal) compared to those in the parallel rising stable bubbles.

The second pool process correlation used in SPARC is one relating the initial globule volume to the vent type and vent Weber number.

$$
W e=\rho_{p} D_{0} V_{0} 2 / \gamma
$$

where

$$
\begin{aligned}
& P_{\mathrm{p}}=\text { pool liquid density } \\
& D_{0}=\text { Dool liquid surface tension } \\
& V_{0}=\text { exit velocity of the gas }
\end{aligned}
$$

In SPARC it is assumed that $Q=V_{0} \pi D_{0} 2 / 4$, where $Q$ is the gas volumetric flow rate at the vent in equilibrium with pool conditions at the vent depth. The correlation used relates the normalized globule volume $v_{n}$ to the Weber number

$$
v_{n}=a w^{b}
$$

where

$$
D g=\frac{3}{2} \nu_{n} D_{0}^{2}\left(\gamma / \rho_{p} g\right)^{1 / 2}
$$


Correlation constants used in SPARC are:

\begin{tabular}{|c|c|c|c|}
\hline Vent & $\mathrm{a}$ & b & Source \\
\hline $\begin{array}{l}\text { - Multiple small } \\
\text { holes }\end{array}$ & 3.45 & 0.46 & EPRI program (Paul et al. 1985) \\
\hline - Downcomer & 0.0891 & 0.616 & $\begin{array}{l}\text { PNL using EPRI data (Paul, } \\
\text { Newman, and Cudnik 1986) }\end{array}$ \\
\hline - Horizontal vent & 0.857 & 0.73 & $\begin{array}{l}\text { EPRI program (Paul, Newman, and } \\
\text { Cudnik 1986) }\end{array}$ \\
\hline
\end{tabular}

These correlations only apply to inlet gases containing noncondensible gases. Very high steam fractions provide for residual bubbles. High steam fractions have a "cone" shaped region that does not detach from the vent.

\section{Stable Bubble Size and Shape}

The stable bubble swarm has a bubble size distribution that is essentially lognormal (Paul et al. 1985). This size distribution remains constant throughout the rise period because of the frequent coalescence and redispersion of bubbles. Although it would be desirable to treat all bubbles according to the swarm statistics (most frequent $\mathrm{d}_{B}(\mathrm{a})=0.57 \mathrm{~cm}$ with a standard deviation of 0.186$)$, the SPARC -90 code currently uses a single diameter to represent the swarm. This diameter is $0.72 \mathrm{~cm}$ (for only noncondensible gas entering at the vent exit), which is the volume mean diameter $\left(d_{v m}\right)$ defined by

$$
d_{v m}=\left(\sum_{i} \frac{n_{i} d_{i}}{N}\right)^{1 / 3}
$$

where $n_{i}$ is the number of bubbles of diameter $i$ in a flow cross-sectional volume, then

$$
N=\sum_{i} n_{i}
$$

When steam accompanies noncondensible gases entering at the vent exit, the effective $d_{v m}$ decreases. The effect of steam can be represented by the expression (developed from the data of Paul et al. 1985)

$$
d_{v m}=0.72 \exp \left\{2.303\left[-0.2265+\left(0.0203+0.0313 x_{n c}\right)^{1 / 2}\right]\right\}
$$

(a) equivalent sphere diameter. 
where $X_{n c}$ is the mole fraction of noncondensible gas in the gas entering the pool.

The shape of the $d_{v m}$ bubble (or any bubble in a swarm) is calculated using another correlation from the data of Paul et al. (1985).

$$
\frac{b}{a}=0.84107+1.13466 d_{v m}-0.3795 d_{v m}^{2}
$$

This correlation was established for $0.15 \mathrm{~cm} \leq \mathrm{d}_{\mathrm{vm}} \leq 1.3 \mathrm{~cm}$. Values of $\mathrm{d}_{\mathrm{vm}}$ outside this range will give incorrect b/a vaTues. All bubbles smaller than $0.15 \mathrm{~cm}$ are spheres $(\mathrm{b} / \mathrm{a}=1.0)$, and bubbles larger than $1.3 \mathrm{~cm}$ have $\mathrm{b} / \mathrm{a}=$ 1.47 in swarms only. Isolated, single, large bubbles can be much flatter and can become lenticular in shape when rising.

\section{Bubble Rise Velocity}

The relative velocity $\left(V_{r}\right)$ of the rising bubble and the water phase is a very important parameter (see Figure 2.2). The present choice of correlations for $V_{r}$ comes from data from Haberman and Morton (1953) and suggested correlations from Zuber and Findlay (1965). The correlations used in SPARC-90 are for equivalent sphere diameter of bubbles, $d_{B}$

$$
\begin{aligned}
& v_{r}\left(d_{B} \leq 0.5 \mathrm{~cm}\right)=7.876(\gamma / p)^{1 / 4}(\mathrm{~cm} / \mathrm{s}) \\
& v_{r}\left(d_{B}>0.5 \mathrm{~cm}\right)=1.40713 v_{r}\left(d_{B} \leq 0.5 \mathrm{~cm}\right) d_{B}^{0.49275}(\mathrm{~cm} / \mathrm{s})
\end{aligned}
$$

where $\boldsymbol{\gamma}$ and $\rho$ are pool surface tension (dyne/ $\mathrm{cm}$ ) and density $\left(\mathrm{g} / \mathrm{cm}^{3}\right)$, respectively. Equation (2.17) is not true for $\mathrm{dB}<0.15 \mathrm{~cm}$. Few bubbles in the swarm are $<0.15 \mathrm{~cm}$ and $\mathrm{d}_{\mathrm{vm}}>0.6 \mathrm{~cm}$.

\section{Swarm Rise Velocity}

The residence time $\left(t_{b}\right)$ of bubbles in the pool is determined by the swarm rise velocity $\left(V_{s w}\right)$, and vent depth $\left(h_{p}\right)$. This residence time is an important parameter [see Equation (2.2)] and is determined by

$$
t_{b}=\int_{0}^{h_{p}} d x / v_{s w}
$$


where $x$ is the vertical coordinate from the vent exit to the pool surface. $V_{\text {SW }}$ is not constant along $x$. The volumetric flow rate of the swarm increases as it rises and $V_{S W}$ increases. Also, $V_{S W}$ represents the volumetric average velocity on a cross section of the swarm. Bubbles in the center rise faster than swarm periphery bubbles. Using data from Paul et al. (1985) and General Electric Company (1981), the following correlation was developed for $V_{S W}$ :

$$
V_{S W}=\left[\left(\dot{Q}_{S}+5.33\right) / 3.011 E-03\right]^{1 / 2}[1-3.975 E-04 x](\mathrm{cm} / \mathrm{s})
$$

where $Q$ is the total gas volumetric flow rate $(\ell / s)$ at depth $h_{p} / 2, \mathrm{~cm}$. In SPARC-90 an average swarm velocity is used:

$$
\bar{v}_{s}=\left[v_{s}\left(h_{p}=0\right)+v_{s}\left(h_{p}=\text { vent depth/2) }\right]\right.
$$

Using this average value simplifies calculations considerably.

\subsubsection{Aerosol Capture}

The SPARC-90 code contains the aerosol capture models for the vent exit region (diffusiophoretic or steam condensation, impaction, and centrifugal deposition) and for the swarm rise region (sedimentation, centrifugal deposition, and Brownian diffusion).

\section{Vent Exit Centrifugal Deposition Models}

SPARC-87 uses an empirical model for centrifugal deposition during gas injection. This model is based on a best fit with data for two empirical constants $A_{1}$ and $A_{2}$ in the depletion of $n_{j}$ particles of size " $i$ " in a bubble

$$
d n_{i}=-n_{i} V_{s_{i}} A_{1} V_{e}^{A_{2}}
$$

or

$$
D F_{i}=\exp \left(V_{S_{i}} A_{1} V_{e}^{A_{2}}\right)
$$

where $V_{S}$ is the settling velocity of size $i$ and $V_{e}$ is vent exit gas velocity. The best fit $A_{1}$ and $A_{2}$ are 18.56 and 0.512 , respectively (if $V_{s_{i}}$ and $V_{e}$ are
in $\mathrm{cm} / \mathrm{s}$ ). Also, see Section 5 . 
SPARC-90 uses a more mechanistic approach for centrifugal deposition during gas injection. This mechanism is important for the large $V_{e}$ expected about the vent injection bubbers. The bases for the model are assumptions Both velocity and geometry control the

The assumptions abob

injection globule is spherical geometry detachment are that the final detached the forming globule is elongated wiameter $\mathrm{Dg}$ and during globule formation, (opening diameter) moving at velocity $v_{0}$ relaspherical front of diameter $D_{0}^{\prime}$

where the velocity becomes

$$
V_{b}=V_{b o} /\left(1+t / \tau_{0}\right)
$$

where $V_{b o}$ is the detachment velocity and $\tau_{0}$ is a characteristic stopping time
as defined below.

$$
\tau_{0}=\frac{\rho_{g} D_{g}}{3 f \rho_{L} V_{0}}
$$

where $f$ is a friction factor (SPARC-90 uses 0.2$),$ and $\rho g$ and $\rho L$ are gas and
liquid densities, respectively.

A subroutine (DFVENT) calculates the usual centrifugal deposition velocity for the two steps. The injection and after detachment and arrives at DFS

$$
D F_{i}(\text { injection })=\exp \left(V_{C i} / V_{0}\right)
$$

for particle size $i$.

Likewise, the DF after detachment is

$$
D F_{i} \text { (detached glosule) }=\exp \left(\frac{V_{0} V_{g i} \rho_{g}}{9 D_{0} f g \rho_{L}}\right)
$$

$V_{g i}$ is the gravitational deposition velocity defined in Equation (2.38).
This DFi is considerably smaller than the injection DF 


\section{Vent Exit Diffusional Deposition}

SPARC-90 calculates the Brownian diffusion contribution to particle capture using the film penetration theory for both injection and afterdetachment regimes. Define $t_{f}$ as the filling time of the globule volume $\nu_{\mathrm{g}}$. Then the diffusional DFs are:

$$
D F_{i}(\text { injection })=\exp \left[\frac{16}{3 D_{0}}\left(\frac{D_{i} t_{f}}{\pi}\right)^{1 / 2}\right]
$$

and

$D F_{i}($ detached globule $)=\exp \left\{\frac{12}{D_{0}}\left(\frac{D_{i}}{\pi D_{0}}\right)^{1 / 2} \frac{1}{a}\left[\left(a t^{*}+\frac{1}{V_{0}}\right)^{1 / 2}-\left(\frac{1}{V_{0}}\right)^{1 / 2}\right]\right\}(2.29)$

Here $t^{*}=3 \tau_{0}$, an assumption and

$$
\begin{aligned}
& a=\frac{3}{4}\left(\frac{\rho_{\mathrm{g}}}{\rho_{\mathrm{D}}}\right) \frac{\mathrm{f}}{\mathrm{D}_{\mathrm{g}}} \\
& \mathrm{D}_{\mathrm{g}}=\left(\frac{6 \nu_{\mathrm{gO}}}{\pi}\right)^{1 / 3}
\end{aligned}
$$

The corresponding DFs for gravitational settling are

$$
D F_{i}(\text { injection })=\exp \left(\frac{A_{s} v_{g i} t^{\hbar}}{\nu_{g o}}\right)
$$

and

$$
D F_{i}(\text { detached })=\exp \left(\frac{3}{2 D g} v_{g i} t^{*}\right)
$$


where $A_{S}$ is the settling area of $1 / 2$ globule (as a bullet shape) during injection, i.e.,

$$
A_{S}=2 \frac{\nu_{g o}}{\pi D_{0}}+D_{0}^{2}\left(\frac{\pi}{8}-\frac{1}{6}\right)
$$

\section{Steam Condensation}

During globule breakup at the vent exit, the equilibration of vapor can remove particles from the gas if net vapor condensation occurs. The simplest model is used here. The fraction of particles removed equals the fractional loss in gas volume caused by condensation at the temperature and pressure of the pool at vent depth. This is easily expressed as DFec:

$$
D F_{\text {ec }}=x_{0} / x_{i}
$$

where $X_{j}$ is the mole fraction of noncondensible gas in inlet gas and $X_{0}$ is the mole fraction of noncondensible gas in the gas after it attains thermal and vapor equilibrium in the pool at the inlet depth. $X_{j}$ is an input value at any given time. $X_{0}$ can be easily related to the pool vapor pressure $\left(P_{W}\right)$, pressure above the pool (PT), and vent depth $\left(h_{p}\right)$ by

$$
x_{0}=1-P_{W} /\left(P_{T}+\rho_{L} g h_{P}\right)
$$

If $X_{0}<X_{i}$, then $D F e c=1$, because $D F$ cannot be less than 1 by definition.

\section{Impaction}

To complete current vent exit models, a set of impaction equations was added to an early version of SPARC -90 by $K$. H. Lee of Battelle Columbus Laboratories. These equations (listed below) were developed from data presented in Fuchs (1964). If gas leaves the exit vent at a high velocity, the initial globules rapidly lose that velocity. The forward globular interface, as it slows and stops, can capture particles if they have sufficient inertia. Inertia of particle size $i$ is represented by the Stokes number $\left(\right.$ Stk $\left._{i}\right)$ :

$$
\text { Stk }_{i}=\rho_{i} v_{e} d_{i}^{2} / 18 \mu l
$$


where $d_{j}=$ particle diameter $(\mathrm{cm})$

$\begin{aligned} \rho_{i} & =\text { particle density }\left(\mathrm{g} / \mathrm{cm}^{3}\right) \\ V_{e} & =\text { vent exit gas velocity }(\mathrm{cm} / \mathrm{s}) \\ \ell & =\text { characteristic length }(0.5 \mathrm{~cm} \text { is a typical orifice radius) } \\ \mu & =\text { gas viscosity (poise). }\end{aligned}$

The DF for this impaction process is

$$
\mathrm{DF}_{\mathrm{I} i}=1 /\left(1-\alpha_{j}\right)
$$

where

$$
a_{i}=1.79182(3.3437 \mathrm{E}-11)(5.9244 \mathrm{E}-03)^{\mathrm{Stk}_{i}}{ }^{1 / 2}
$$

if Stk $_{j}^{1 / 2} \leq 0.65888$ and

$$
a_{i}=1.13893(1.4173 \mathrm{E}-06)(4.25973 \mathrm{E}-03)^{\mathrm{Stk}_{i}}{ }^{1 / 2}
$$

if $0.658686<\operatorname{Stk}_{j} 1 / 2<1.4$. For $\operatorname{Stk}_{j} 1 / 2>1.4, a_{j}=0.99$. Although these are included in SPARC-90, their importance is minimal unless near-sonic values of $v_{e}$ occur. Other possible vent exit scrubbing mechanisms are discussed in Section 2.1.3.

\section{Gravity Settling During Swarm Rise}

For gravity settling, centrifugal deposition, and Brownian diffusion, the corresponding deposition velocities $V_{g}, V_{C}$, and $V_{D}$ are now defined. Starting with $V_{g}$, the deposition velocity follows Stokes law [which applies to spherical particles $<70-\mu m$ aerodynamic equivalent diameter (AED)]:

$$
v_{g i}=\rho_{p} d_{i}^{2} g C n_{i} / 18 \mu
$$

where subscript $i=$ particle size $i$

$\mathrm{Cn}_{i}=$ Cunningham slip correction factor (subroutine DIFFU)

$d_{j}=i$ size particle diameter

$\mu=$ gas viscosity. 
For particles $>70 \mu \mathrm{m} A E D$, a set of empirical equations are used to find $V_{g i}$ (Knudsen 1970). These equations are found in the D0 704 loop in SPARC-90. To accommodate bubble geometry and make $V_{g}$ perpendicular to the bubble surface, $V_{g i}$ is multiplied by $\cos (\beta)$ (see Figure 2.2).

\section{Centrifugal Deposition During Swarm Rise}

Bubbles larger than a critical size exhibit surface circulation as they rise through liquid (Calderback and Lochiel 1964). Fuchs (1964) developed the equation for circulation in spherical bubbles. SPARC-90 models circulation in oblate-spheroidal bubbles.

The pioneering work in the centrifugal scrubbing of oblate-spheroidal bubbles was done by Demitrack and Moody (1983). The essential equations are reported here in cylindrical polar coordinates instead of the elliptical coordinates of the original authors. The development of circulation velocity ( $V_{S}$, Figure 2.2) or the bubble surface is essential to obtaining centrifugal forces acting on particles in the bubble near the gas surface. The equation for local surface velocity is:

$$
v_{s}=v_{r}\left[\left(1-B_{3} A\right) / E_{c 2}-v_{r} B_{3}\right] B_{5} /\left(B_{3}^{2}+B_{6}^{2}\right)^{1 / 2}
$$

where $V_{r}=$ bubble rise velocity [Equations (2.17) and (2.18)]

$a=$ ellipse major axis (see Figure 2.2)

$b=$ ellipse minor axis

$\theta=$ cylindrical polar coordinate $(\pi / 2 \leq \theta \leq-\pi / 2)$

$E_{C 1}=$ eccentricity $=[1-(b / a) 2] 1 / 2$

$E_{C 2}=E_{C 1}\left(1-E_{C 1}\right) 1 / 2=\sin ^{-1}\left(E_{C 1}\right)$

$A=\tan \left(1 / B_{3}\right)$

$c=\left(a^{2}-b^{2}\right) 1 / 2$

$r=$ radial coordinate $=\left[1 /\left(B_{2}+B_{1}\right)\right]^{1 / 2}$

$B_{1}=(\cos \theta / a)^{2}$

$B_{2}=(\sin \theta / b)^{2}$

$B 3=b / c$

$B 4=a / c$

$B 5=r \cos \theta / a$

$B_{6}=r \sin \theta / b$
$r_{C}=$ surface radius of curvature $=c\left(B_{3}^{2}+B_{6}^{2}\right)^{3 / 2} /\left(B_{4}+B_{3}\right)$. is

The centrifugal capture velocity of a particle of size $i$ at the surface

$$
v_{c i}=v_{s}^{2} v_{g i} / r_{c} g
$$




\section{Brownian Diffusion During Swarm Rise}

Aerosol particles have a diffusion coefficient that can be calculated using the Stokes-Einstein Equation (Fuchs 1964):

$$
\left.D_{i}=k T C_{m i} / \pi d_{i} \mu\right)
$$

where $D_{i}$ is the diffusion coefficient for particle size $i$, and $k$ is Boltzmann's constant.

The local deposition velocity, VDi, from Brownian diffusion can be estimated from penetration theory of mass transfer (Bird, Stewart and Lightfoot 1960; Crank 1967):

$$
V_{D i}=\left(\frac{D_{i}}{\pi t_{e}}\right)^{1 / 2}
$$

where $t_{e}$ is the exposure time of the moving surface. At the top of the bubble, $t_{e}=0.0$. Equation (2.42) holds when no vapor is crossing the interface simultaneously with the particles. However, as bubbles rise, vapor enters the bubble to maintain vapor equilibrium. The vapor flux depends on pool temperature, bubble parameters, and various transport coefficients. Equation (2.42) can be adjusted to accommodate $V_{V}$, the vapor velocity. A corrected $V_{D i}$ becomes

$$
V_{D i}^{\prime}=\xi_{i} V_{D i}
$$

where

$$
\xi_{i}=\exp \left(-\phi_{i}^{2}\right) /\left[2-\exp \left(-1.85 \phi_{j}\right)\right]
$$

and the parameter $\phi_{i}$ is equal to $V_{v} / V_{D i}$.

\section{Overall DF}

All quantities have now been defined to obtain an overall DF; for each particle size $i$ from the instant of gas-to-water contact to bubble destruction at the pool surface. The vector addition of all deposition velocities (except Voil) minus the vapor velocity is

$$
v_{n i}=v_{c i}+v_{g i} \cos \beta-v_{v}
$$


where $\beta=$ angle designated in Figure 2.2. Here

$$
\beta=\tan ^{-1}\left[b^{2} / \tan (\theta) a^{2}\right]
$$

If $V_{n i} \leq 0$, then $V_{n i}$ is set equal to zero and the net deposition velocity is

$$
V_{n i}^{\prime}=V_{D i}^{\prime}
$$

If $V_{n i}>0$ in Equation (2.45),

$$
v_{n i}^{\prime}=v_{n i}+v_{D i}^{\prime}
$$

Next $V_{n j}$ must be integrated over the entire bubble surface to get the overall $\mathrm{DF}_{i}$ in time step $\Delta t$ :

$$
D F_{i}=\exp \left[2 \pi \Delta t \int_{\theta=\pi / 2}^{-\pi / 2} r \cos (\theta) V_{n}^{\prime} d s / \frac{4}{3} \pi a^{2} b\right]
$$

where ds is the differential of arc length of the $a-b$ ellipse. The cumulative $\mathrm{DF}\left(\overline{\mathrm{DF}}_{j}\right)$ for the entire process for each particle size $j$ is then the product of the series of DFs, i.e,..:

$$
\overline{D F}_{j}=D F_{e c} D F_{I j}\left[D F_{j}(1) D F_{j}(2) \ldots . D F_{j}(N)\right]
$$

where $N$ is the number of rise steps taken at each $\Delta t$, and where $N \Delta t$ is $h_{p} / V_{S w}$.

\subsubsection{Thermal Hydraulics}

This section discusses models used to describe the thermodynamic behavior of rising bubbles and the transfer of vapor and energy across the bubble walls. 


\section{Bubble Thermodynamics}

The thermodynamic states of the bubbles as they rise from the vent exit to the pool surface must be described to estimate particle growth. The beginning assumption in the rise of the bubbles is that the initial bubbles are in thermodynamic equilibrium with the pool at the vent exit depth $h_{p}$. As the bubble rises an incremental distance from depth $x$ (state 1) to $x-d x$ (state 2), the bubble can expand because of the drop in pressure $\left(P_{2}-P_{1}\right)$ and influx of water vapor.

$$
P_{1}=P(x)=P_{T}+\rho_{P} g x
$$

and

$$
P_{2}=P(x-d x)=P_{T}+\rho_{p} g(x-d x)
$$

Also during the $d x$ change

$$
\begin{aligned}
T_{2} & =T_{1}+d T \\
M_{v 2} & =M_{v 1}+d M_{v}
\end{aligned}
$$

where $M_{V}$ is the $g$-moles of water vapor in the bubble.

Next we define the thermodynamic state of the bubble (internal energy) at each position. The basis for this state is zero internal energy at $273.2 \mathrm{~K}$, $1 \mathrm{~atm}$ for liquid water.

$$
U_{1}=M_{v 1} \lambda_{v}+\int_{273.2}^{T}{ }^{T}\left[M_{v 1} C_{v v}+M_{n c} C_{v n c}+m_{\ell} C_{\ell}^{\prime}+m_{s} C_{s}^{\prime}\right] d T
$$

and similarly for $U_{2}$ where only $T_{2}$ and $M_{v 2}$ differ from $T_{1}$ and $M_{v 1}$.

Here $\quad \lambda_{V}=$ internal energy of evaporation at $273.2 \mathrm{~K}, 1 \mathrm{~atm}(\mathrm{~J} / \mathrm{g}$-mole)

$M_{n c}=g$-moles of noncondensible gas in bubble

$m_{\ell}=$ mass of condensed water as aerosol particles in bubble gas

$m_{S}=$ mass of solids as aerosol particles in bubble gas

$C_{V v}=$ heat capacity of water vapor at constant volume (J/g-mole/K)

$C_{v n c}=$ heat capacity of noncondensible gases at constant volume

$C_{l}=$ heat capacity of liquid water, $\mathrm{J} / \mathrm{g} / \mathrm{K}$

$\mathrm{C}_{\mathrm{s}}^{\prime}=$ heat capacity of solids, $\mathrm{J} / \mathrm{g} / \mathrm{K}$. 
The intake of new water vapor $d M_{v}$ is calculated from rate equations at state 1 conditions where both temperature and steam gradients exist.

To find $T_{2}$, the first law of thermodynamics must be used:

$$
d U=U_{2}-U_{1}=d Q-\delta W
$$

Here dQ is the differential heat added to the bubble and is calculated according to Equation (2.66). SW is the differential work of expansion in $d x$ done by the bubble from pressure drop, vapor production, and temperature change:

$$
\delta W=R\left[M_{t} T_{1} \ln \left(\frac{P_{1}}{P_{2}}\right)+\left(T_{2}-T_{1}\right) M_{v 1}+T_{1} d M_{v}\right]
$$

where $d M_{v}=M_{v 2}-M_{v 1}$ and $M_{t}=M_{v 1}+M_{n c}$. With $\bar{C}_{v v}$ and $\bar{C}_{v n c}$ as average values of heat capacity over the range of temperature 273.2 to $T_{1}, K$, Equation (2.56) can be rewritten to give $T_{2}=A / B$ as the only unknown. Thus

$$
\begin{aligned}
A=\{ & d Q-R T_{1}\left[d M_{v}+M_{t} * \ln \left(P_{1} / P_{2}\right)-M_{t}\right]-d M_{v} \lambda_{v} \\
& +\bar{C}_{v n c} M_{n c} T_{1}+\bar{C}_{v v}\left(M_{v 1} T_{1}+273.2 d M_{v}\right) \\
& \left.+C_{\ell}^{\prime} T_{1} m_{l}+c_{s}^{\prime} T_{1} m_{s}\right\}
\end{aligned}
$$

and

$$
B=\left[R M_{T}+C_{v n c} M_{n c}+c_{v v} M_{v z}+C_{\ell}^{\prime} m_{\ell}+C_{s}^{\prime} m_{s}\right]
$$

The above treatment does not allow dme grams of water vapor to condense on particles in $d x$, so $T_{2}$ must be adjusted to $T_{3}$. This is done by an iterative method (DO 205 loop in SPARC) to define the new $T_{3}$ and dme and $d M_{v}^{\prime}=d M_{v}-18 d m_{\ell}$. The process of correcting $T_{2}$ to $T_{3}$ involves the equations for vapor pressure, gas saturation, and mass balances. The iterative process continues until the gas saturation level is 1.0 (100\% relative humidity). The value of dme calculated this way is the thermodynamic maximum amount. Section 2.2.4 examines the rate limiting maximum, dme. 


\section{Bubble Heat and Mass Transfer}

Heat and mass are transferred into a rising bubble bringing the bubble toward the equilibrium temperature and vapor pressure of the pool. The model used in SPARC-90 to estimate this heat and mass transfer again involves penetration theory (see Section 2.2.2).

Both heat and mass transfer rates depend on three temperatures, the pool temperature, Tp; the bubble temperature, Tb; and the local interface

temperature, $T_{s}=T_{s}(r, \theta)$. The incremental sensible heat transfer into the bubble from the bulk pool liquid to the bubble interface at any $r, \theta$ over time step $d t$ is

$$
\mathrm{dQ}_{1}=\left(\mathrm{T}_{\mathrm{p}}-\mathrm{T}_{\mathrm{s}}\right) \mathrm{dq}_{1}
$$

where

$$
\mathrm{dq}_{1}=\rho_{\mathrm{p}} \mathrm{C}_{\ell}^{\prime} \mathrm{dAdt}\left(a_{w} / \pi t_{e}\right)^{1 / 2}
$$

from penetration theory as in Equation (2.42). Here the differential surface element $d A=2 \pi r d s$, where $d s=r \cos \theta d \theta ; a_{w}=$ thermal diffusivity of liquid water; and $C_{l}^{\prime}$ is the water heat capacity, $\mathrm{J} / \mathrm{g} / \mathrm{K}$. The sensible heat transfer into the bubble by conduction in the gas/vapor phase is

$$
\begin{aligned}
& d Q_{2}=\left(T_{s}-T_{b}\right) d q_{2} \\
& d Q_{2}=\left(T_{s}-T_{b}\right) \rho_{b} C_{p b} d A d t\left(a_{b} / \pi t_{e}\right)^{1 / 2}
\end{aligned}
$$

where $C_{p b}=$ heat capacity of the bubble at constant pressure and $a_{b}=t_{1}$ ermal diffusivity of the bubble. The latent heat added to the bubble is

$$
\mathrm{dQ}_{3}=\lambda_{\mathrm{p}} \mathrm{dM} \mathrm{M}_{\mathrm{v}}
$$

where $\lambda_{p}$ is the enthalpy of vaporization, $\mathrm{J} / \mathrm{g}$-mole. The heat balance across the interface is

$$
\mathrm{dQ}_{3}+\mathrm{dQ}_{2}=\mathrm{dQ}_{1}
$$


which can be solved for $T_{s}$ :

$$
T_{s}=\left(-d Q_{3}+d q_{2} T_{b}+d q_{1} T_{p}\right) /\left(d q_{1}+d q_{2}\right)
$$

Then the heat quantity in Equation (2.56) is

$$
\mathrm{dQ}=\mathrm{at} \int_{-\pi / 2}^{\pi / 2} \mathrm{dQ} \mathrm{Q}_{1}
$$

The simultaneous mass transfer estimate using penetration theory is developed in a manner similar to the heat transfer estimate. The goal here is to calculate $d M_{v}, g$ moles water vapor entering the bubble in $d t$. Because $T_{s}$ $>T_{b}$, the vapor pressure of water, $P_{v s}$, at the interface is greater than that of the bubble, Pvb. The corresponding water vapor mole fractions at the interface, $x_{s}$, and in the bubble, $x_{b}$, are

$$
\begin{aligned}
& x_{s}=P_{s} / P_{2} \\
& x_{b}=P_{b} / P_{2}
\end{aligned}
$$

The driving force for mass transfer through a stagnant film with a constant $X_{S}$ is (Bird, Stewart and Lightfoot 1960)

$$
\chi=\left(x_{s}-x_{b}\right) /\left(1-x_{s}\right)
$$

and from penetration theory the local steam flux at $r, \theta$ into the bubble is

$$
N_{s}=\chi\left(\frac{P_{2}}{R T_{s}}\right)\left(\frac{D_{s}}{\pi t_{e}}\right)^{1 / 2}
$$

This flux should be corrected for high mass transfer rates. The method of Bird, Stewart and Lightfoot (1960) is used, and the correction factor $\Omega$ is

$$
\Omega=\log _{e}(\chi+1) / \chi
$$


The value of $d M_{v}$ then can be calculated as

$$
d M_{v}=d t \int \Omega N_{s} d A=d t \int_{=\pi / 2}^{\pi / 2} 2 \pi r \chi \Omega\left(\frac{P_{2}}{R T_{s}}\right)\left(\frac{D_{s}}{\pi t_{e}}\right)^{1 / 2} d s
$$

This integral is performed numerically in SPARC-90.

\subsubsection{Particle Growth, SPARC-87}

Particles can grow or shrink by water condensation or evaporation when saturation levels of the surrounding gas change. Soluble particles have a unique effect on the growth behavior, i.e., they can keep water in the particle even in subsaturated atmospheres. The SPARC-87 approach considered the soluble effects separately from the growth possible in supersaturated atmospheres.

\section{Soluble Particles}

Soluble hygroscopic particle growth is explained in the document describing the prototype code (Owczarski, Postma and Schreck 1985). Here the use of the model is discussed to simulate soluble particle growth. Additional discussion is found in Owczarski, Schreck and Winegardner (1985), which is paraphrased below.

Although not independent in reality, because of ease of computation and prior development, the soluble particle-growth model is considered independently from growth in supersaturated atmospheres. The first assumption is that soluble substances in the particles are dissolved in water and are in equilibrium with a subsaturated $(S<1.0)$ atmosphere. Growth is frozen at $S$ $=0.99$ because of this phenomenon. When $S>1.0$, growth proceeds as described in the next section, but with the initial diameter equal to the equilibrium particle diameter at $S=0.99$. This cutoff value of $S=0.99$ is somewhat arbitrary. Only experimental comparison of growth phenomena with soluble and insoluble particles going from low $S$ values to $S>1$ will produce a better number. The second assumption here is that the bubble swarm is equilibrated to pool vapor pressure and temperature at the vent exit.

The equilibrium drop size reached in a humid atmosphere is governed by the degree to which the vapor pressure of water is lowered by the soluble material and the degree to which curvature affects an increase in the vapor pressure. Both effects are well understood and are calculable using classical physics and chemistry. The equilibrium saturation ratio, $S$, is related to drop size by an equation presented by Fletcher (1962). 


$$
S=\frac{\left[\exp \frac{2 \sigma}{n_{L} k T r}\right]}{\left[1+\frac{\operatorname{ImMw_{O}}}{\operatorname{Mw}\left(\frac{4}{3} \pi r^{3} \rho-m\right)}\right]}
$$

where

$$
\begin{aligned}
S & =\text { saturation ratio (the relative humidity) } \\
\sigma & =\text { surface tension of solution (dyne } / \mathrm{cm}) \\
\mathrm{nL}_{\mathrm{L}} & =\text { number of molecules } / \mathrm{cm}^{3} \text { of solution (solvent }+ \text { solute) } \\
r & =\text { radius of drop }(\mathrm{cm}) \\
\mathrm{I} & =\text { van't Hoff ionization factor } \\
\rho & =\text { density of solution (g/cm } 3) \\
M w & =\text { molecular weight of solute }(\mathrm{g} / \mathrm{g}-\mathrm{mole}) \\
M w_{O} & =\text { molecular weight of solvent }(\mathrm{g} / \mathrm{g}-\mathrm{mole}) \\
\mathrm{m} & =\text { mass of solute in the droplet }(\mathrm{g}) .
\end{aligned}
$$

Equation (2.73) was evaluated under the assumption that the solute was cesium hydroxide, the solvent was water, and the temperature was $100^{\circ} \mathrm{C}$.
Results are summarized in Table 2.2 .

The data of Table 2.2 illustrate that particle growth factors depend on relative humidity, $S$, and that significant growth factors are predicted.

\section{Growth in Supersaturated Atmospheres}

A supersaturated atmosphere exists in many cases when cooling of near-saturated gas occurs. To discuss this phenomena quantitatively, $S$, the
degree of saturation, is defined as

$$
S=P_{v} / P_{v}^{0}
$$

where $P_{v}$ is the actual vapor pressure of the water in the gas and $P_{V}^{0}$ is the normal vapor oressure of water at the pressure and temperature of the gas.

TABLE 2.2. Growth of $\mathrm{CSOH}$ Particles in Humid Atmosphere at
$100^{\circ} \mathrm{C}$ with $\mathrm{I}=2$

Dry Particle Radius, $\mu \mathrm{m}$

Droplet Radius in Stated Humidity

$\underline{S=0.9} \quad \underline{S=0.95} \quad \underline{S=0.99} \quad \underline{S=0.999}$

$$
\begin{aligned}
& 0.01 \\
& 0.10 \\
& 1.0 \\
& 10.0
\end{aligned}
$$

$\begin{array}{ll}0.0195 & 0.0225 \\ 0.195 & 0.255 \\ 1.95 & 2.55 \\ 19.5 & 25.5\end{array}$

0.0295

0.425

4.45

44.5

0.0345

0.775

9.35

95.5 
For $S<1$, the gas is subsaturated. When $1<S<4$, supersaturated vapors can condense on heterogeneous wettable surfaces. When $S>4$, water vapor can also condense by homogeneous droplet nucleation and growth.

The rate of heterogeneous particle growth is of primary concern to the depletion of particles from the gas phase. In the flow regime of the bubble swarm, two rate processes limit particle growth. The first process is the rate of cooling of the rising bubbles [see deveiopment of Equation (2.59)]. The second process is the condensation rate on individual particles themselves. This latter rate-limiting step is considered next.

The classical rate equation, called Mason's equation, for growth of droplets in rain clouds is found in the fundamental theories of cloud physics; it is derived in Byers (1965) as

$$
\left.r \frac{d r}{d t}=(S-1) /\left[M w_{v} \Delta H_{v}^{2} \rho_{L} / k g R T^{2}\right)+\left(\rho_{L} R T / P_{v}^{0} D M w_{v}\right)\right]
$$

where $\quad r=$ droplet radius $(\mathrm{cm})$

$t=$ time (s)

$M w_{v}=$ molecular weight of the vapor $(18 \mathrm{~g} / \mathrm{g}$-mole $)$

$\Delta H_{V}=$ latent heat of vaporization $(\mathrm{J} / \mathrm{g})$

$\rho L=$ density of condensed liquid $\left(\mathrm{g} / \mathrm{cm}^{3}\right)$

$\mathrm{kg}=$ gas thermal conductivity $(\mathrm{J} / \mathrm{cm} / \mathrm{s})$

$\mathrm{R}=$ gas constant

$T=$ absolute temperature (K)

$D=$ vapor diffusivity in gas $\left(\mathrm{cm}^{2} / \mathrm{s}\right)$.

Equation (2.75) is written more simply as

$$
r \frac{d r}{d t}=\frac{S-1}{a+b}
$$

and is used in its integrated form (for constant T, S, a and b) (a) as

$$
r^{2}=r_{0}^{2}+\frac{2(S-1)}{a+b}\left(t-t_{0}\right)
$$
(a) The Kelvin restriction prevents particles smaller than diameter d' from
growing (Adamson 1976):

$$
d_{0}^{\prime}=4 \sigma \tilde{V} / R T \ln (S)
$$

where $\tilde{V}$ is the liquid molar volume, $\mathrm{cm}^{3} / \mathrm{g}$-mole. 
Mason's equation accounts for both the diffusive resistance of the condensing vapor as well as the conductive resistance of the gas surrounding the droplet that inhibits the removal of the latent heat of vaporization. The above equations are used in SPARC-90 along with empirical relationships for the temperature-dependent constants $a$ and $b$. Use of Equation (2.77) reveals that all the supersaturated water vapor available can be condensed in less time than the usual time step $d t$ or $\Delta t$ used in SPARC-90. Therefore, the use of Equation (2.78) is only valid when $S$ does not change in $\Delta t$, which can only be true if few aerosol particles exist. In SPARC-90 the thermodynamic growth limit is used when $S$ is not constant and is discussed below.

The rationale and algorithms for calculating particle growth using the thermodynamic limit are discussed in detail by Owczarski, Schreck and Winegardner (1985) for the ICEDF code. This discussion is paraphrased here for SPARC. The calculation of the thermodynamic limiting amount of water that can condense on particles, dme $g$-moles/bubble ( $\Delta$ me in the finite difference calculation of SPARC-90) is discussed in Section 2.2.3. A simple criterion is used in SPARC to determine which limit applies to the situation in the bubble. The Mason's rate equation limit $\Delta m_{\ell} j$ for each particle size $i$ is compared to the thermodynamic limit $\Delta \mathrm{me}_{i}$. The smaller of the two depicts the limiting process for particle size $i$. However, experience shows that only one limiting process applies at each time step for all sizes.

To calculate $\Delta m_{\ell} j$ and $\Delta m_{\ell} i$, Mason's equation (2.76) is integrated over $\Delta t$ at constant $S$ to acquire

$$
18 \Delta m_{\ell j}^{\prime}=2 \pi d p_{j}(S-1) \rho_{L} \Delta t /(a+b)
$$

where $\mathrm{dp}_{\mathrm{i}}=$ particle diameter before $\Delta t$.

If $n_{j}$ is the number of particles of size $i$ in the bubble, then

$$
\Delta m_{\ell}^{\prime}=\sum_{i=i^{\prime}}^{20} n_{i} \Delta m_{\ell i}^{\prime} \quad \text { for } 20 \text { bins }
$$

The index $i$ ' represents the index of the smallest particle that can grow according to the Kelvin restriction.

Because $\quad \Delta m_{\ell}^{\prime}=\sum_{i=i^{\prime}}^{20} n_{i} \Delta m_{\ell i}^{\prime}$ 
must also hold, to apportion the various $\Delta \mathbf{m}^{\prime} i$ on the appropriate particle by Equation (2.78),

$$
18 \Delta m_{\ell}=2 \pi(S-1) \rho_{L} \Delta t /(a+b) \sum_{j=i^{\prime}}^{20} n_{j} d p_{j}
$$

where $S$ is constant but unknown.

Equation (2.81) is then

$$
18 \Delta m_{l}^{\prime}=A \sum_{i=i^{\prime}}^{20} n_{i} d p_{i}
$$

and thus

$$
\Delta m_{l i}^{\prime}=A d p_{i}
$$

where

$$
A=18 \Delta m_{\ell}^{\prime} / \sum_{i=j^{\prime}}^{20} n_{i} d p_{i}
$$

The expression for the constants $a$ and $b$ in Equation (2.78) are estimated by approximations of curves found in Byers (1965).

$$
\begin{aligned}
& a=\rho_{L} \exp \left[13.5008-1.11063 E-02\left(T_{b}-273.2\right)\right] \\
& b=\rho_{L} \exp \left[13.7102-6.45 E-02\left(T_{b}-273.2\right)\right] P_{T} / 1000
\end{aligned}
$$

where PT equals total gas pressure in millibars.

\subsubsection{Particle Growth, SPARC-90}

SPARC-90 combines all particle growth mechanisms into one set of relationships. The result is a simpler mechanistically correct approach. The work of Jokiniemi (1990) has provided two useful bases for SPARC-90. The first is the method for calculating the activity of water in non-ideal solutions of $\mathrm{CSI}$ and $\mathrm{CSOH}$, the two most prominent hygroscopic compounds in nuclear accident aerosols. The second is the assurance that a modified Mason's equation is suitable for nuclear aerosols. The models discussed below are adapted from Jokiniemi (1990). 
The modified Mason's equation used in SPARC-90 replaces the quantity (S - 1) in Equations (2.75) through (2.77) rith $\left(S-S_{r}\right.$ ) where $S_{r}$ is the saturation ratio at a particle surface. For a spherical solution droplet surrounding insoluble matter

$$
S_{r}=A_{l} \exp \left[\frac{2 \sigma_{e} M_{w}}{r R \rho_{l} T}\right]
$$

where $A \ell$ is the activity of the water solution and the exponential quantity is the Kelvin vapor pressure correction for droplets of radius $r$ and surface tension $\sigma$. Equation (2.87) is another way of writing Equation (2.73).

However, to generalize Equation (2.73) to multicomponent systems, we expand Al

$$
A_{l}=\frac{1}{1+\sum_{i} I_{i} n_{i} / n_{w}}
$$

where $I_{j}=v^{\prime}$ an't Hoff ionization factor for solute $i$

$n_{i}=$ moles solute $i$ in droplet

$n_{W}=$ moles water in droplet.

Previously we assumed ideal ionization ( $\left.\mathrm{I}_{i}=2\right)$ for $\mathrm{CsI}$ and $\mathrm{CsOH}$ ( Table 2.2). This is a severe limitation. Using data from Jokiniemi (1990) we have arrived at the formulas

$$
\begin{aligned}
& \mathrm{I}\left(\mathrm{CsOH}, 25^{\circ} \mathrm{C}\right)=1.75467+20.7974 \mathrm{n}(\mathrm{CsOH}) / \mathrm{nT} \\
& \mathrm{I}\left(\mathrm{CsI}, 25^{\circ} \mathrm{C}\right)=1.79417-3.34363 n(\mathrm{CsI}) / \mathrm{nT} \\
& \quad \text { or } \mathrm{n}(\mathrm{CsI}) / \mathrm{nT} \leq 0.021 \\
& I\left(\mathrm{CsI}, 25^{\circ} \mathrm{C}\right)=1.63439+4.30022 \mathrm{n}(\mathrm{CsI}) / \mathrm{nT} \\
& \text { for } \mathrm{n}(\mathrm{CsI}) / \mathrm{nT}>0.021 .
\end{aligned}
$$

These are corrected for temperature using $\mathrm{NaOH}$ data in Jokiniemi (1990), which gives

$$
I\left(T^{\circ} \mathrm{C}\right)=I(25)[1-2.321 \mathrm{E}-03(T-25)]
$$

SPARC-90 uses $I_{j}=2$ for all solutes other than $C S I$ or $C$ SOH as a default value. 
SPARC-90 uses the integral form of Mason's equation

$$
r^{2}=r_{0}^{2}+2 \frac{S-S_{r}}{a+b}\left(t-t_{0}\right)
$$

for small time steps $\left(t-t_{0}\right)$, while it uses the $t_{0}$ values of $S_{r}$ to compute Equation (2.93). This method appears to be satisfactory as long as

$$
2\left(s-S_{r}\right)\left(t-t_{0}\right) /(a+b) \ll r_{0}
$$

because $S_{r}$ is very sensitive to changes in $r$, especially for $r<0.1$ micrometers. 


\subsection{SPARC-90 DESCRIPTION}

This chapter provides details of SPARC-90 organization, calculational flow, and subroutines used. The SPARC-90 version discussed here is the seventh developed to date. The coding of SPARC began as a collection of particle deposition models for spherical bubbles from Fuchs (1964). The Fuchs spherical bubble equations were modified to approximately accommodate the elliptical (oblate spheroidal) bubble models of Demitrack and Moody (1983). After adding a model for the influx of steam in rising bubbles, the code was released as a prototype of SPARC-90 (Owczarski, Postma and Schreck 1985). Later versions had an improved steam influx model, a more rigorous application of the elliptical bubble model, and detailed thermodynamic descriptions of the rising bubble including the expansion work of rising bubbles. This expansion work causes the bubbles to cool to supersaturated conditions where the aerosol particles grow by condensation. Particle growth can increase the DF of the pool by orders of magnitude under certain pool conditions, aerosol size distributions, and aerosol concentrations.

\subsection{CODE ORGANIZATION}

Because SPARC-90 evolved from a small simple code, it is not 'structured' in the popular trend. It does, however, use a number of supporting subroutines. The main code could be rewritten in the 'structured' sense, but the few potential advantages do not justify the effort.

The primary function of SPARC-90 is to compute DFs for two regions of the bubble swarm: the vent exit or swarm formation region and the swarm rise region. All other operations and algorithms support this function. Figure 3.1 is a flow chart of the main program in SPARC-90. A brief discussion of each block in the flow chart follows.

\section{1 .1 Read Input File}

The input data are read in using a free-field format. These data consist of pool conditions, aerosol particle properties, inlet gas composition and conditions, inlet particle and carrier gas mass flow rates, and SPARC-90 calculational and output parameters. All but the control parameters are entered as discrete variables at discrete time values during an accident.

\subsubsection{Write Input File}

SPARC-90 echoes the input file as its first output. Each input variable is labeled by the variable name. 


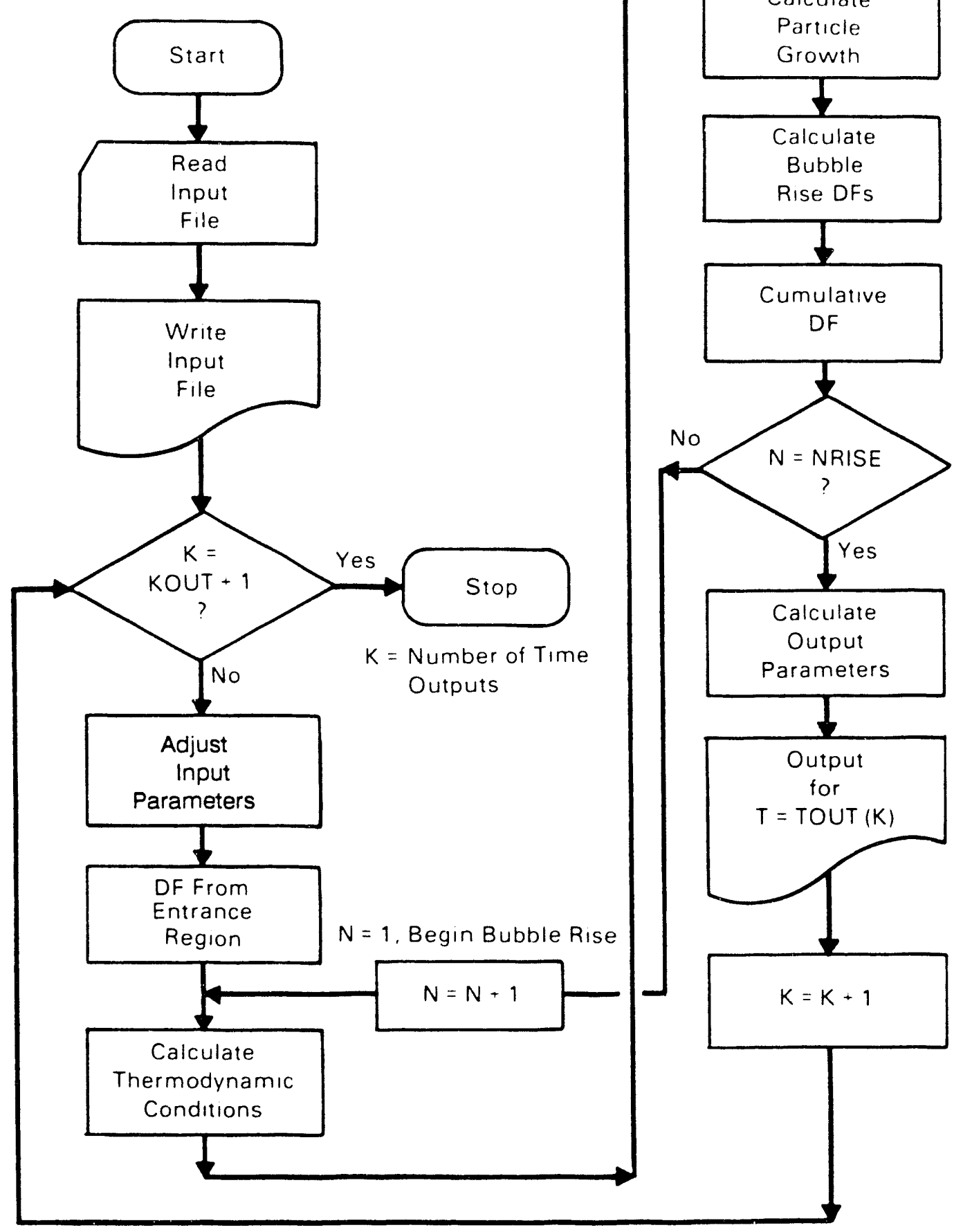

FIGURE 3.1. Flow Chart of the SPARC-90 Code 


\section{1 .3 Output DO Loop}

The user has specified the number (KOUT) of output times that DF calculations are to be made. At a corresponding set of times, TOUT $(K=1$, ... KOUT), SPARC-90 interpolates all changing input conditions to correspond to each TOUT(K) and follows a bubble of aerosol from the vent inlet to the top of the pool.

\subsubsection{Input Adjustment}

First the code considers the appropriate TOUT(K) value to interpolate between time-dependent variables specified in the input. Then the inlet gas conditions are appropriately defined for determining particle-removal rate constants for DF calculations.

\subsubsection{DF Entrance Region}

Gases and particles enter the pool at a specified depth $h_{p}$ from vents. SPARC-90 computes the scrubbing of particles at this point via four mechanisms (Section 2.2.2): condensation of steam, inertial impaction if vent exit velocities are high, centrifugal deposition, and Brownian diffusion. Bubbles are brought to equilibrium conditions at $h_{p}$ and particle growth on soluble particles is computed.

\subsubsection{Swarm Rise DO Loop}

The swarm rise calculations begin by following a bubble for NRISE discrete steps from depth $h_{p}$ to the top of the pool. The DO $500 N R=1$, NRISE loop begins with the aerosol conditions at the gas entrance region after the calculations described in Section 3.1.5 are complete. After each NR loop is completed, the bubble is returned to its original diameter.

\subsubsection{Thermodynamic Conditions}

At the beginning of each NR loop, the bubble thermodynamic conditions are calculated for the new bubble position in the pool. First the amounts of heat and vapor transferred into the bubble are calculated [Equations (2.59) and (2.72) ] and then the First Law of Thermodynamics is invoked to get a new bubble temperature, $T_{b}$, without new condensation on particles (Section 2.2.3). Finally, the thermodynamic maximum possible condensation ( $\Delta \mathrm{m}_{\ell}$ ) is calculated along with a corrected $T_{b}$.

\subsubsection{Particle-Growth Calculations}

In this code section, newly condensed water vapor is distributed on the airborne particles by either using a thermodynamic limit or a growth rate limit method (SPARC-87). SPARC-90 uses Equation (2.77) with a modified droplet vapor pressure [Equation (2.87)]. 


\subsubsection{Bubble Rise DF Calculations}

The DF during bubble rise is calculated using the three deposition mechanisms and the hindering influx of vapor. The DF during bubble rise for each particle size $i$ is DFbr:

$$
D F_{i}^{b r}=\prod_{N=1}^{N R I S E} D F_{i}(N)
$$

where each $D F_{i}$ is calculated by Equation (2.49).

\subsubsection{Cumulative DF}

This cumulative $\overline{D F}_{j}$ is the product of the entrance region $D F s$ and the bubble rise DFbr for each particle size.

$$
\overline{\mathrm{DF}}_{i}=\mathrm{DF}_{\text {ec }} \mathrm{DF}_{\mathrm{Ii}} \mathrm{DF}_{i}^{\mathrm{br}}
$$

The overall DF (DFOv) for all particle sizes is the ratio of particle mass flow rate into the pool/particle mass flow rate out of the pool. We define $\hat{P}_{\mathrm{mi}}$ and $\hat{P}_{\mathrm{mo}}$ as particle mass flow rates of size $i$ particles into and out of the pool, respectively. Then

$$
D F^{O V}=\sum_{i=1}^{20} \dot{p}_{m i} / \sum_{i=1}^{20} \dot{p}_{m o}
$$

or

$$
D^{\text {OV }}=\sum_{i=1}^{20} \dot{\mathrm{P}}_{\mathrm{mi}} / \sum_{i=1}^{20} \dot{\mathrm{P}}_{\mathrm{mi}} / \overline{\mathrm{DF}}
$$

The value of DFOV is the most important output value calculated by SPARC-90 for accident analyses. 


\subsubsection{Output Parameter Calculations}

Output printing is scheduled for each TOUT (K=1, .., KOUT). The first output preparation consists of listing six variables at pool exit conditions as functions of the particle bin number $\mathrm{J}=1, \ldots, 20$ : particle diameters, both dry and wet; particle flow rate in $\mathrm{g} / \mathrm{s}$, both dry and wet; particle numbers; and decontamination factor. Then 18 other quantities are prepared including number of median particle radii (wet and dry), particle geometric standard deviation, and overall DF [Equation (3.4)]. See Section 4.3 for an output example.

\subsection{CALCULATIONAL SUBROUTINES}

The subroutines below provide calculations for the main program.

\subsubsection{Subroutine DIFFU}

This subroutine computes the Brownian diffusion coefficient, $D_{j}$, in $\mathrm{cm}^{2} / \mathrm{s}$ as a function of particle diameter, $d_{j}$, the mean free path of gas molecules, $\lambda$, in $\mathrm{cm}$, and the Cunningham slip factor, $C_{j}$. As developed in Fuchs (1964)

$$
\begin{gathered}
D_{j}=1.38 E-16 T C n_{j} /\left(3 \pi \mu d_{j}\right), \mathrm{cm}^{2} / \mathrm{s} \\
C n_{j}=1+2.492 \frac{\lambda}{d_{j}}+0.84 \frac{\lambda}{d_{j}} \exp \left(-0.435 \frac{d_{j}}{\lambda}\right), \text { dimensiontess } \\
\lambda=1.245 E-02(T / M w)^{0.5} / P
\end{gathered}
$$

where $P$ equals absolute gas pressure (atm).

\subsubsection{Subroutine KELVIN}

This subroutine determines whether particles can grow under the restrictions of the Kelvin equation (see footnote, p. 2.26). Growth is restricted if the vapor pressure of a curved droplet exceeds that of the surrounding vapor.

\subsubsection{Subroutive I2EQUIL}

This subroutine calculates the I2 equilibrium coefficient HEQ for the gas-water interface. HEQ is the equilibrium $I_{2}$ concentration in the liquid/I2 concentration in the gas. 


\subsubsection{Subroutine SOLGRO}

This subroutine calculates the equilibrium particle diameter for hygroscopic particles for SPARC -87 .

\subsubsection{Subroutine REQUIL}

This subroutine replaces SOLGROW for SPARC-90.

\subsubsection{Subroutine PSI2ABS}

This subroutine allows the user to react $\mathrm{I}_{2}$ with $\mathrm{CsOH}$ particles in the primary system.

\subsubsection{Subroutine PGROW}

This subroutine calculates the dynamic growth of hygroscopic particles not in equilibrium with gas water vapor (SPARC-90 only).

\subsubsection{Subroutine DFVENT}

This subroutine calculates SPARC-90 centrifugal deposition and Brownian diffusion in the vent injection region. 


\subsection{CODE OPERATION AND PARAMETER SELECTION}

SPARC-90(87) is written in FORTRAN. The input file uses the free format of FORTRAN 77. This chapter details the necessary input requirements of the code and describes the output along with examples of both input and output. Guidance for choosing parameters is given in Sections 4.1.1 and 4.1.2.

\subsection{INPUT REQUIREMENTS}

The input fiie of SPARC-90 requires three types of information: 1) pool physical parameters, 2) inlet aerosol parameters, and 3) calculational and output specifications. In addition, the user must specify the version of SPARC to be used, by using 1990 or 1987 for the input variable IVERS.

\subsubsection{Pool Physical Parameters}

For detailed descriptions of specific suppression pools, the reader is referred to the Safety Analysis Reports published during the licensing process for each reactor. The suppression pool information required in SPARC-90 is not very detailed. The input variables required are

$\begin{array}{rll}\operatorname{POOLT}(J)= & \text { pool temperature }\left({ }^{\circ} \mathrm{C}\right) \mathrm{J}=1, \text { NDATA } \\ \text { POOLP }(J)= & \begin{array}{l}\text { pressure above the pool, absolute atmospheres, } J=1, \text { NDATA } \\ \text { number of data entry points per run where input parameters are }\end{array} \\ \text { TI }(J)= & \begin{array}{l}\text { specified } \\ \text { time values (minutes) of each data entry point } J=1, \text { NDATA }\end{array}\end{array}$

\subsubsection{Inlet Aerosol Size Distribution Parameters}

The parameters needed include both inlet particle characteristics as well as inlet bulk gas characteristics. Because inlet particle sizes and flow rates are probably the most important parameters in SPARC-90, considerable effort should be made to specify these parameters throughout the accident sequence. Up to 20 particle sizes are specified with the NBINS variable. These NBIN sizes can be a discrete representation of a particle-size distribution. The example below represents a NBINS $=20$ lognormal distribution around a mass median diameter (MMD) of 1.0 E-04 cm $(1 \mu \mathrm{m})$. That DPART (NBINS) vector combined with a vector for $\operatorname{ZMASS}(\mathrm{J}, \mathrm{I})$, which is the percent of PMSDOT(J) in bucket size I at time $J$, where the latter is the particle dry mass flow rate entering the pool.

$$
\begin{array}{ll}
\operatorname{ZMASS}(1, I) & =0.0160 \\
\operatorname{ZMASS}(2, I) & =0.0590 \\
\operatorname{ZMASS}(3, I) & =0.195 \\
\operatorname{ZMASS}(4, I) & =0.530 \\
\operatorname{ZMASS}(5, I) & =1.50 \\
\operatorname{ZMASS}(6, I) & =3.30 \\
\operatorname{ZMASS}(7, I) & =5.90 \\
\operatorname{ZMASS}(8, I) & =9.50 \\
\operatorname{ZMASS}(9, I) & =14.0 \\
\operatorname{ZMASS}(10, I) & =15.0
\end{array}
$$

$$
\begin{aligned}
& \operatorname{ZMASS}(11, I)=15.0 \\
& \operatorname{ZMASS}(12, I)=14.0 \\
& \operatorname{ZMASS}(13, I)=9.50 \\
& \operatorname{ZMASS}(14, I)=5.90 \\
& \operatorname{ZMASS}(15, I)=3.30 \\
& \operatorname{ZMASS}(16, I)=1.50 \\
& \operatorname{ZMASS}(17, I)=0.530 \\
& \operatorname{ZMASS}(18, I)=0.195 \\
& \operatorname{ZMASS}(19, I)=0.0590 \\
& \operatorname{ZMASS}(20, I)=0.0160
\end{aligned}
$$


This input completes an inlet particle-size distribution at time TI(I) with a geometric standard deviation, $\sigma_{\mathrm{g}}=2$, and a total inlet particle concentration of $5 \mathrm{E}-06 \mathrm{~g} / \mathrm{cm}^{3}$ at the inlet gas conditions. To change the MMD by a certain factor at input time TI(I), specify the factor by DMULT(I). If the user wishes to change $\sigma_{g}$, each ZMASS (J, I) must be changed. It is easy to set $\sigma_{g}$ to $\sigma_{g}=$ $\infty$ where each $\operatorname{ZMASS}(\mathrm{J}-1, \mathrm{I})=\operatorname{ZMASS}(\mathrm{J}, \mathrm{I})=\operatorname{ZMASS}(\mathrm{J}+1, \mathrm{I})=5$ and to $\sigma_{\mathrm{g}}=1$ where only one $\operatorname{ZMASS}(\mathrm{J}, \mathrm{I})=100$. The DPART(I) input vector can easily be changed for this purpose, too.

The requirements for the carrier gas description are:

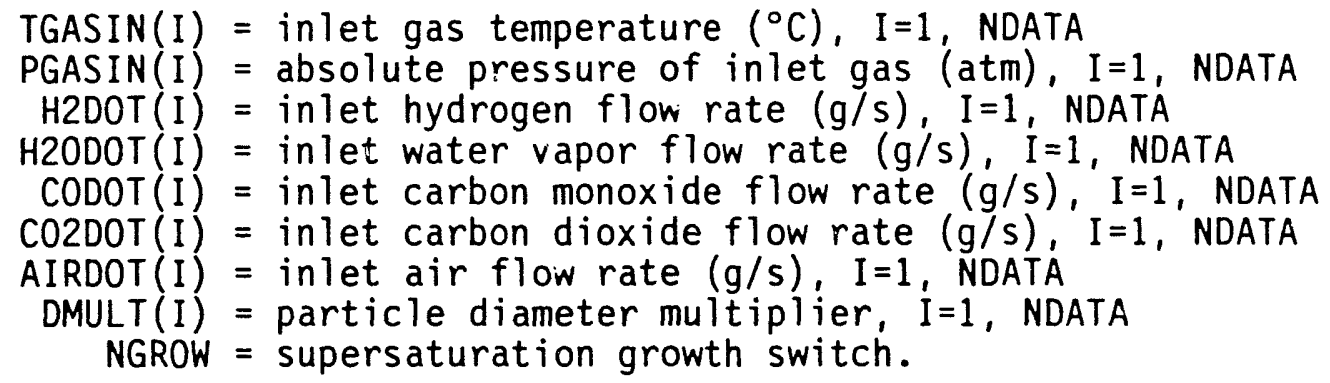

If the user wants to suppress supersaturation growth of particles during bubble rise, set NGROW $\neq 1$.

\subsubsection{Vent Parameters}

The user has three types of vent choices. Only one vent type can be selected per SPARC run. If multiple vent types are operating during an event, the types must be run separately with flows apportioned accordingly. The variable selecting vent type is MVENT. MVENT = 1 for multiple quenchers ( $X$, $T$, or PWR); MVENT $=2$ for downcomers; and MVENT $=3$ for horizontal vents. If MVENT $=1$, the number of holes in the multihole quencher is NVENT. If MVENT $\neq 1$, NVENT $=1$. The number of MVENTS is NTYPE. The submergence $(\mathrm{cm})$ of each type is SUBXT, SUBDC, and SUBHV. The cross-sectional area of each vent $\left(A_{x}\right)$ is represented by DVENT $=\left(A_{x}\right)^{1 / 2}, \mathrm{~cm}$. The enclosed sample problem illustrates the use of these parameters (see Section 4.3).

\subsubsection{Aerosol Species and Other Chemical Species Parameters}

Some aerosol species and other chemical species caused by the gas require specifying certain information.

For aerosol particles, these include:

$$
\begin{aligned}
\text { RHOI }(I)= & \text { insoluble particle density, } g / c C, I=1, \text { NDATA } \\
\text { RHOS (I) = } & \text { soluble particle density, } g / c C, I=1 \text {, NDATA } \\
\text { VHI (I) }= & \text { van't Hoff ionization factor for soluble salts } \\
& \text { (SPARC-87 uses this. Any number will do for SPARC-90), } \\
& \text { I=1, NDATA }
\end{aligned}
$$


AMWS $(I)=$ average molecular weight of soluble material, I=1, NDATA

SOLF (I) = dry weight fraction of soluble material, I=1, NDATA

PMSDOT $(I)=$ particle mass flow rate into pool, $g / s, I=1$, NDATA

$\operatorname{FRCS}(\mathrm{I})=\mathrm{CsOH}$ mass fraction in PMSDOT(I), I=1, NDATA

$\operatorname{FRRB}(\mathrm{I})=\mathrm{RbOH}$ mass fraction in $\operatorname{PMSDOT}(\mathrm{I}), \mathrm{I}=1$, NDATA.

For iodine species, these include:

D0TI2 $(\mathrm{I})=\mathrm{I} 2$ mass flow rate, $\mathrm{g} / \mathrm{s}$ into pool, $\mathrm{I}=1$, NDATA

ORIDOT(I) = organic iodide mass flow rate, $g / \mathrm{s}$ into pool, $I=1$, NDATA

FRPI(I) = weight fraction of soluble iodide in PMSDOT(I)

JOD: If JOD $=1$, all vapor iodine species scrubbing in pool is off

MPS: If MPS $=1$, subroutine PSI2ABS is called to absorb I2 in primary system.

\subsubsection{Calculational and Output Specifications}

Only four input numbers are required in this category: 1) NRISE, the number of calculational steps during bubble rise; 2) NCIRC, the number of surface area increments on the bubble surface; 3 ) KOUT, the desired number of times of output; and 4) TOUT ( $K, K=1, K O U T)$ minutes (see Section 3.1.3.).

NRISE has some control over accuracy of calculations. Larger values will give smaller rise increments to the rising bubbles. Usually this should mean that the rise, $\overline{D F}_{i}$, should become more accurate as NRISE increases.

Experience has shown that NRISE $>20$ is desirable, but NRISE $>200$ may use unnecessary computer time. Another guideline is to keep $10 \mathrm{~cm}>\mathrm{HPD} / \mathrm{NRISE}>$ $1 \mathrm{~cm}$.

NCIRC also has some control over the accuracy of calculations. This value divides the surface of the bubble into NCIRC by dividing the angle $\theta$ (in Figure 2.2) into $\pi /$ NCIRC radians. Experience has shown that NCIRC $=20$ seems adequate. Larger NCIRC might increase accuracy slightly, but at the expense of computer time.

KOUT and TOUT ( $K, K=1, K O U T$ ) are the output controls. KOUT must be $>1$ and TOUT $(1)=0.0$. No other restrictions are on KOUT and TOUT(K) as long as KOUT $\leq 100$ (DIMENSION statement limit) and TOUT (KOUT) $\leq$ TI (NDATA).

\subsubsection{Order of Input Values}

SPARC is programmed by subroutine INTRO to prompt the user to name the input file, e.g., NAME1.INP. The subroutine then creates the output file NAME1.OUT. If NAME1. INP is available, the code will commence execution. ERROR messages will appear if the input file is incorrectly ordered.

To make a correctly ordered file, the input variable must appear in the order specified by the 29 READ statements in subroutine INPUT (see the Appendix). Each READ statement requires a new row of input data, each datum 
followed by a comma, except the last datum, according to the free format used. Each of the 29 READ statement variables are now discussed below (see Table 4.1 for an example).

READ1,

(1st): An alphanumeric title in the first 80 spaces

(2nd): IVERS, NDATA, NBINS in the first 72 spaces (each row following must be in the first 72 spaces or on the very next row).

(3rd): $\quad$ TI (I), I $=1$, NDATA

(4th): RHOI(I), "

(5th): RHOS (I), "

(6th): VHI(I), "

(7th): AMWS(I), "

(8th): $\quad$ SOLF (I), "

(9th): PMSDOT(I),"

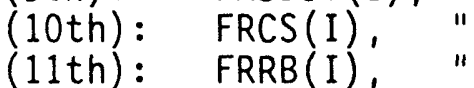

(12th): $\operatorname{DPART}(\mathrm{I}), \mathrm{J}=1$, NBINS

(13th): This read statement requires a row for $\operatorname{ZMASS}(J, I), I=1, \operatorname{NDATA}$ each $\mathrm{J}=1$, NBINS

(14th): H2DOT(I), I=1, NDATA

(15th): H2ODOT(I), "

(16th): CODOT(I), "

(17th): CO2DOT(I),"

(18th): AIRDOT(I),"

(19th): DOTI2(I), "

(20th): ORIDOT(I), "

(21st): FRPI(I), "

(22nd): TGASIN(I), "

(23rd): PGASIN(I),"

(24th): PODLT(I),"

(25th): POOLP(I)," "

(26th): DMULTI(I), "

(27th): KOUT, VPOOL, KLVN, NGROW, DX, NVENT, DVENT, MVENT, NTYPE, SUBXT, SUBDC, SUBHV, MPS, JOD

(28th): TOUT $(K), K=1$, KOUT

$(29 \mathrm{th}): \quad$ NCIRC

\subsection{OUTPUT DESCRIPTION}

The current programmed output consists of two parts: the 'echo' of the input file and the output calculated for each time step TOUT(K). The calculated output also consists of two parts: the essential particle-size distribution information at the pool surface, and the bubble parameters and other information in the overall particle distribution and gas conditions. These are described in Sections 4.2 .1 and 4.2 .2 and in examples discussed in 4.3 . 


\subsubsection{Output Description by Particle Size}

The output file per TOUT(K) is headed by a TOUT(K) print and 'Materials Leaving Pool'. Below this is a matrix of seven vertical 20-component vectors of these materials for each particle size:

1. particle bin number

2. particle dry diameter $(\mathrm{cm})$

3. wet particle $(\mathrm{cm})$

4. particle flow rate, dry $(\mathrm{g} / \mathrm{s})$

5. particle mass flow rate, wet $(\mathrm{g} / \mathrm{s})$

6. particle number flow rate (particles/s)

7. particle decontamination factor.

\subsubsection{Additional Output}

For the same TOUT! $(x), 37$ other outlet (unless otherwise noted) variables are printed. These are listed in the tables in the sample problem, located in Section 4.3 .

\subsection{INPUT/OUTPUT EXAMPLES - SAMPLE PROBLEM}

Tables 4.1 and 4.2 are examples of an input file and the output echo of that input file. Tables 4.3 and 4.4 are examples of the output at TOUT(1) $=$ 0.0 for the input file of Table 4.1 .

\subsection{PARAMETER SENSITIVITY STUDY}

This section summarizes parameter sensitivities in SPARC-90. With the summary, the user can concentrate on the most important controlling parameters in specifying code input. The ranking of importance is determined solely by the overall decontamination factor.

A sensitivity study (Owczarski and Postma 1984) ranked the controlling parameters without supersaturated particle growth. A later paper (Owczarski and Postma 1985) included the effects of this particle growth. Table 4.5 was constructed from the two papers to rank the parameters. This ranking is based on a single small orifice vent.

Under certain conditions these rankings may not be maintained. The parameters themselves are not independent of each other. For example, the particle growth mechanism, which operates because of the presence of supersaturation in rising bubbles, has been discovered to be very important, especially at low particle concentration. All of the other parameters interact in this growth mechanism. Present experience shows that the rankings given in Table 4.5 remain true if inlet particle concentrations are $>\sim 3 \mathrm{E}-06 \mathrm{~g} / \mathrm{cm}^{3}$. 


\section{TABLE 4.1. Example of an Input File}

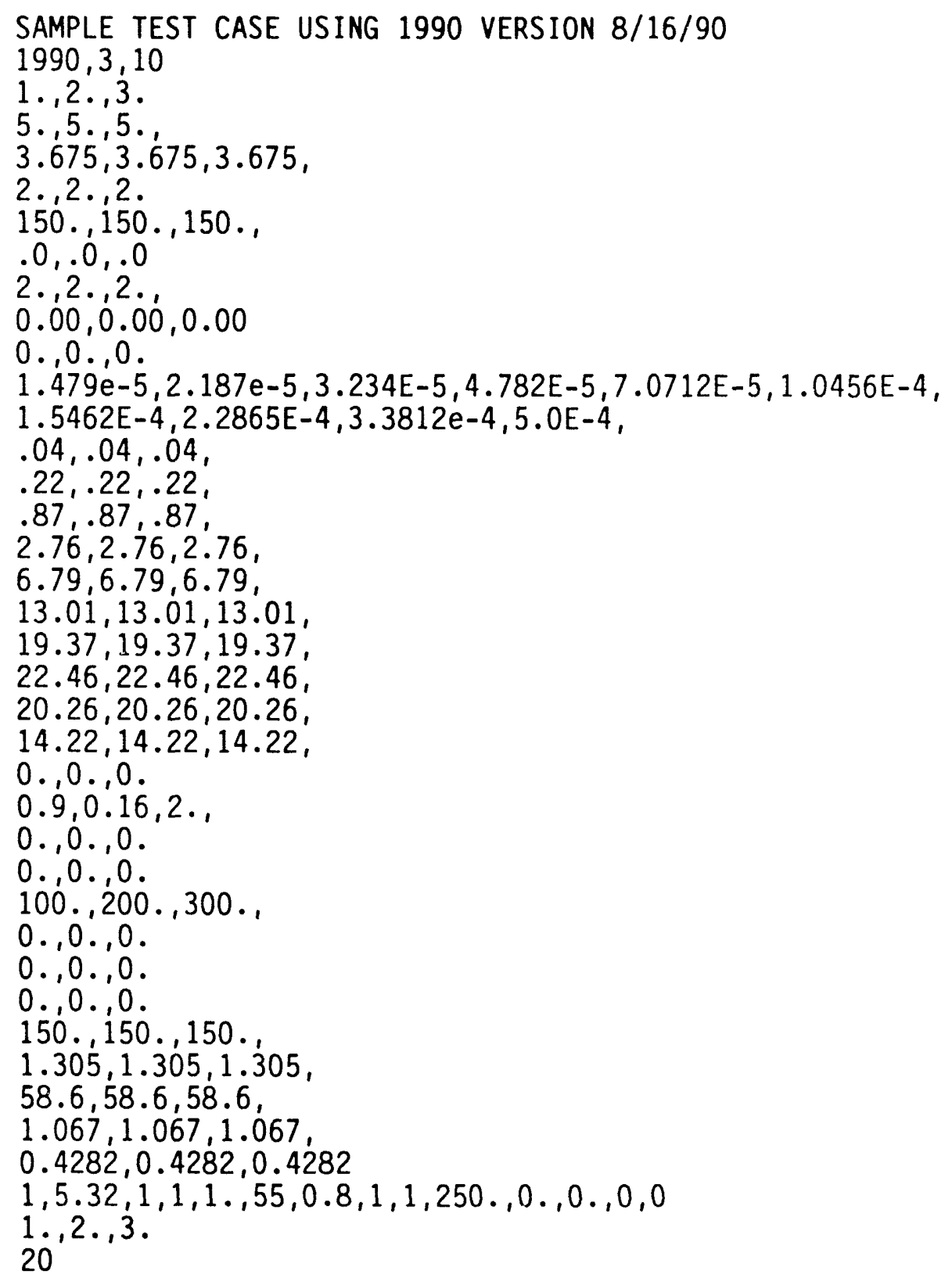


TABLE 4.2. SPARC Printout of Table 4.1 Input File SAMFLE TEST CASE USING 1998 VERSION $8 / 16 / 80$

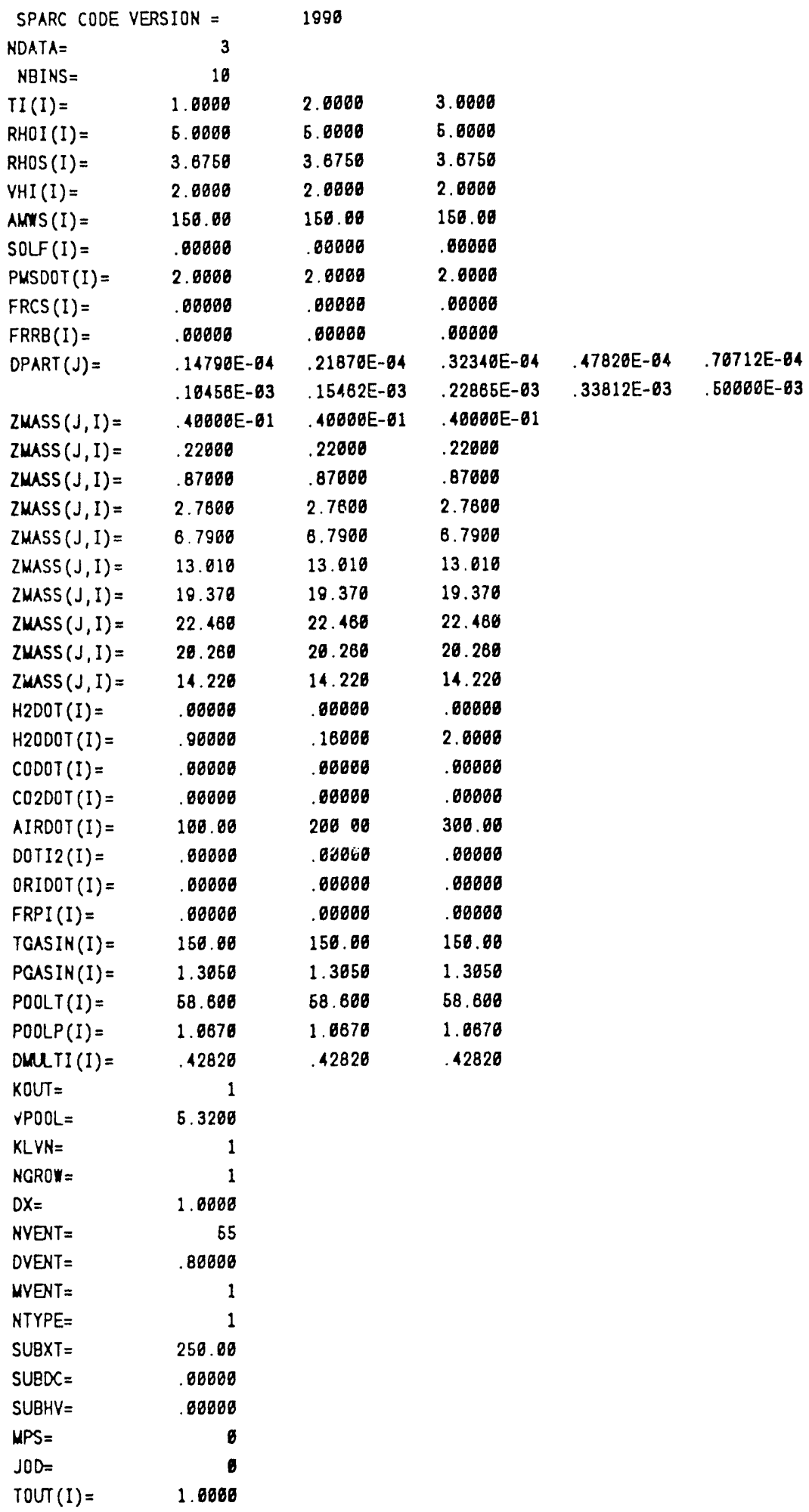


TABLE 4.3. Particle-Size Output Data for Table 4.1 Input File at 0.0 min.

\begin{tabular}{|c|c|c|c|c|c|c|}
\hline PARTICLE & PARTICLE & PARTICLE & GRAMS & GRAMS & NUMBER & DECON. \\
\hline BIN & DIAM & DIAM & PER SEC & PER SEC & PER SEC & FACTOR \\
\hline NUMBER & DRY (CM) & WET (CM) & DRY & MET & & DF \\
\hline 1 & $6.3331 E-66$ & $6.3331 \mathrm{E}-86$ & $6.0532 \mathrm{E}-64$ & $6.6532 E-64$ & $9.1028 E+11$ & $1.3216 \mathrm{E}+\theta 0$ \\
\hline 2 & $9.3647 \mathrm{E}-66$ & $9.3647 \mathrm{E}-86$ & $3.6324 E-63$ & $3.6324 \mathrm{E}-63$ & $1.4103 E+12$ & $1.4516 E+\theta 6$ \\
\hline 3 & 1.3848E- 85 & $1.3848 \mathrm{E}-65$ & $9.7680 E-63$ & $9.7686 \mathrm{E}-63$ & $1.4650 \mathrm{E}+12$ & $1.7813 E+66$ \\
\hline 4 & $2.6477 \mathrm{E}-65$ & $2.6477 \mathrm{E}-85$ & $1.9983 \mathrm{E}-62$ & $1.9983 \mathrm{E}-62$ & $8.8906 E+11$ & $2.7623 E+66$ \\
\hline 5 & $3.6279 \mathrm{E}-65$ & 3.6279E-65 & $1.6355 \mathrm{E}-62$ & $1.6355 \mathrm{E}-62$ & $2.2504 E+11$ & $8.3833 E+\varnothing 6$ \\
\hline 6 & $4.4773 E-65$ & $4.4773 \mathrm{E}-65$ & $1.6385 \mathrm{E}-62$ & $1.6385 \mathrm{E}-62$ & $4.4198 E+10$ & $2.5055 \mathrm{E}+01$ \\
\hline 7 & 6.6268E- -65 & 6.6288E-65 & 6. 2966E-64 & 6. 2966E-64 & $8.2871 E+\varnothing 8$ & $6.1525 E+62$ \\
\hline 8 & $9.7968 \mathrm{E}-65$ & $9.7968 E-65$ & $8.6697 \mathrm{E}-67$ & 8. $6897 E-67$ & $3.5284 E+05$ & $5.1812 E+85$ \\
\hline 9 & $1.4478 E-64$ & $1.4478 E-64$ & $4.7877 \mathrm{E}-13$ & $4.7877 \mathrm{E}-13$ & $6.0257 \mathrm{E}-62$ & $8.4633 \mathrm{E}+11$ \\
\hline 10 & 2.1410E- 4 & $2.1410 E-64$ & $1.6110 \mathrm{E}-26$ & $1.6110 \mathrm{E}-26$ & 6. $2761 E-16$ & $1.7654 E+25$ \\
\hline
\end{tabular}


TABLE 4.4. Overall Aerosol Properties and 0ther Parameters - Part of the Output File for Table 4.1 Input File at $0.0 \mathrm{~min}$.

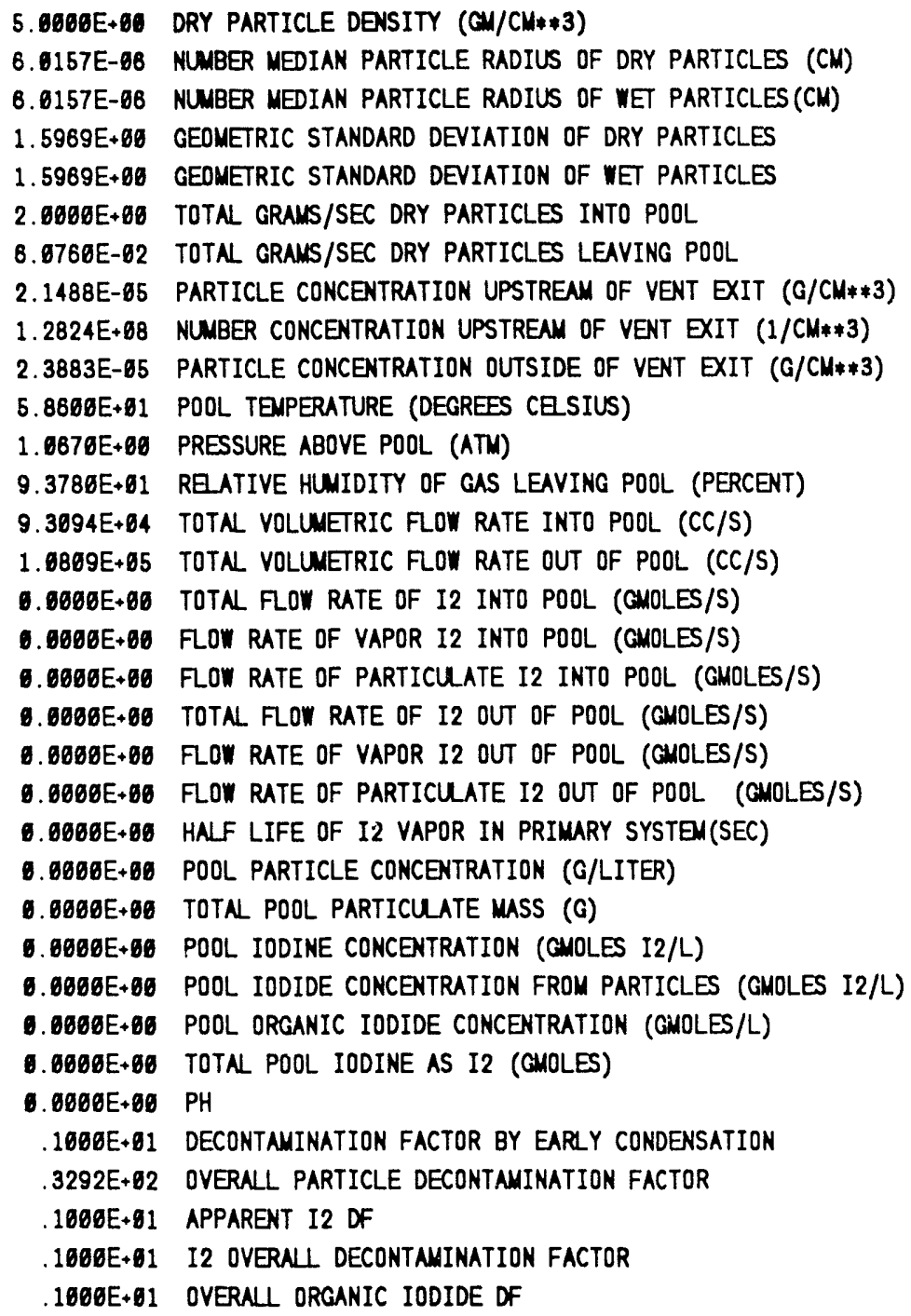

POOL DECONTAMINATION FACTORS INTEGRATED OVER DURATION OF FLOWS FROM TOUT(1) TO TOUT(KOUT)

\author{
3.2917E+61 TIME INTEGRATED PARTICLE DF \\ 1.0000 +00 TIME INTEGRATED I2 DF \\ $1.06606+60$ TIME INTEGRATED ORGANIC IODIDE DF
}


TABLE 4.5. Parameter Sensitivity Summary

Most Important

- Particle size distribution

Very Important

- Particle concentration

- Bubble size/shape

- Volume fraction of steam in inlet gas

- Particle density

Intermediate Importance

- Pool temperature

- Pool depth

- Percent of soluble material in particles

Least Important

- Noncondensible gas composition

- Pressure above pool 


\subsection{MODEL DATA COMPARISONS}

\subsection{INITIAL COMPARISON}

Initial model data comparisons are reported by Owczarski and Winegardner (1985). Underprediction of the extent of particle scrubbing prompted several empirically based modifications to the code. Specifically, in the various models in SPARC-87, six experimental constants are now needed: two for the vent exit scrubbing model, two for a bubble redispersion model, and one each for particle solubility effects and effective bubble size. SPARC-90 eliminates the need for some of these experimental constants.

\section{1 .1 Initial Data Base}

The data base (Cunane et al. 1985) available for initial comparison consists of particle scrubbing measurements taken in a pool using a $0.5-$ in. diameter horizontal injector. The following conditions were varied during 56 different experiments: inert gas composition (air or helium); steam composition; gas flow rate; injector depth; pool temperature (ambient or near boiling); and aerosol ( $C_{S} I, \mathrm{Te}_{2}$, or $\mathrm{Sn}$ ) size, solubility, density, and concentration. Decontamination factor measurements for each experiment consist of the time-integrated particle mass flow rate into the pool divided by timeintegrated particle mass flow rate out of the pool. These DFs are reported along with estimates of experimental uncertainty.

\subsubsection{Comparison Criteria}

The comparison criteria used are based on the agreement between measured and calculated log DFs. The quantitative degree of agreement can be calculated using three quantities for $n$ experiments: standard error (SE), mean difference (MD), and coefficient of correlation ( $\left.R^{2}\right)$. These quantities are defined as

$$
\begin{aligned}
& S E=\left\{\sum_{j}[\log D F(\text { measured })-\log D F(\text { calculated })] j^{2 / n}\right\}^{1 / 2} \\
& M D=\sum_{j}[\log D F(\text { measured })-\log D F(\text { calculated })] j / n \\
& R^{2}=\left[1-\frac{n(S E)^{2}}{\sum_{j}\left[\log D F(\text { measured })-\log D F M j^{2}\right.}\right] 100
\end{aligned}
$$

where $\log D F M=\Sigma \log D F$ (measured) $j / n$. 


\subsubsection{SPARC-87 Models}

Before comparison results are presented, two new models for SPARC are discussed. These models were found to be necessary to explain unacceptable differences between experimental and calculated values. The horizontal injector causes considerable gas momentum to exist in the initial globules, which causes particle scrubbing by inertial deposition from centrifugal forces. During swarm rise, bubbles coalesce and redisperse. The redispersion process also causes curved surface motion resulting in centrifugal force deposition. The new models are discussed below.

For vent exit bubble inertia, the initial globule scrubbing velocity for particle size $i$ is a function of the injection rate. An analysis of globule volume and velocity suggest that the injection DF be of the form

$$
D F_{i}=\exp \left[A_{1} V_{s}(i) V_{e}^{A 2}\right]
$$

where $A_{1}$ and $A_{2}$ are experimental constants, $V_{s}(i)$ is the settling velocity of particle size $i$, and $V_{e}$ is the vent exit velocity. See Section 2.1 for additional discussion of the model.

Similarly, the bubble dispersion model DF can be expressed as

$$
D F_{i}=\exp \left[E V_{s}(i) \gamma \Delta t / d_{b} g \mu\right]
$$

where $E$ is an experimental parameter related to the formation frequency of a bubble, $\gamma$ is the liquid surface tension, $\mu$ is the liquid viscosity, $d_{b}$ is the bubble equivalent volume sphere diameter, $\Delta t$ is the total bubble rise time, and $g$ is the acceleration of gravity. The parameter $E$ depends on the swarm density which, in turn, depends on swarm volume flow rate Gs. In SPARC, an empirical model assumes that

$$
E=A_{3} G_{s} A_{4}
$$

where $A_{3}$ and $A_{4}$ are experimental constants.

\subsubsection{Initial Comparison Results}

Before the development and application of the new models, the first comparison did not look promising. Prototype SPARC DFs were always lower than experimental DFs and often by orders of magnitude (Owczarski, Postma, and Schreck 1985). The initial comparison gave $S E=1.03, M D=0.79$, and $R^{2}$ $=7.22 \%$. The SE value can be interpreted as an average spread factor of 10.6 
and the MD as an under prediction factor of 6.2. Obviously the prototype SPARC model did not account for one or more particle capturc mechanisms.

With the inclusion of two new models, the parameter (experimental constant) optimization process began. This entailed changing one experimental constant at a time until the lowest SE and MD and largest $\mathrm{R}^{2}$ values were obtained for each. This process was repeated once for each constant. A noticeable improvement in agreement was observed. This agreement was reflected in $S E=0.42, M D=0.072$, and $R^{2}=83 \%$. The $S E$ can be interpreted as a spread factor of 2.6 (antilog of SE) and the MD as an underprediction factor of 1.2 (antilog of MD). The high $R^{2}$ seems adequate.

There is an uncertainty in the data that is not reflected in the experimental uncertainty estimates. Too few replicate runs were made to determine the true experimental variance. If all of the data were true, the user of SPARC -87 has an apparent $68 \%$ certainty that the SPARC DF is within a factor of 2.6 of the true DF. Because the data base has noise in itself, one cannot calculate the true uncertainty of the SPARC-87 DF. Without this information, the adequacy of SPARC -87 for use in accident analyses should be judged on its apparent uncertainty relative to the uncertainty of all other calculational methods of the accident analysis.

The other experimental constants that have been obtained indicate that the area mean bubble diameter multiplier (of volume mean bubble diameter) $\bar{d}_{a}$ appears to be the best single size representative of the swarm and that the effect of particle solubility on particle growth is present but less than expected. Values of the six experimental constants are provided here.

$$
\begin{aligned}
A_{1} & =18.56 \\
A_{2} & =0.512 \\
A_{3} & =0.034 \\
A_{4} & =0.5 \\
\text { SATMLT } & =0.88 \\
\bar{d}_{a} & =0.912
\end{aligned}
$$

Only $A_{3}, A_{4}$, and $\bar{d}_{a}$ are used in SPARC -90 .

\subsubsection{SPARC Iodine Scrubbing Comparisons}

No large-scale data were available for iodine vapor species comparisons. However, iodine capture models are validated by small-scale tests. The data of Diffey et al. (1965) compare favorably with SPARC calculations for both I2 and $\mathrm{CH}_{3} \mathrm{I}$. 


\subsection{SPARC -87 COMPARISON}

The topic of this section is the summary of results of comparisons of SPARC-87 with Advanced Containment Experiments (ACE) (a) (multihole) and Battelle Columbus Laboratories (BCL) (courtesy of EPRI, see acknowledgments) (large vents and varying multihole vents) experimental data. The SPARC -87 version has the various vent models (empirical scrubbing and large bubble breakup) in operation. Table 5.1 summarizes the particle scrubbing DFs for the ACE experiments and SPARC calculated DFS (SPARC A and SPARC B corresponding to each available particle size data set). Table 5.2 summarizes the $B C L$ data/SPARC comparison. Data codes in Table 5.2 are: HV $=2-\mathrm{ft}$ diameter horizontal vent; $D C=2-\mathrm{ft}$ diameter downcomer; and $M H=$ multihole spargers $(10,10,4,4,1=$ no. of $0.5-\mathrm{in}$. diameter holes in MH1 to MH5, respectively). Figure 5.1 is a $\log / \log$ plot of the two DF data sets of Tables 5.1 and 5.2 .

Some statistical parameters that show the agreement between SPARC-87 and all the experimental data of the figure are: $M D=0.00531$, $S E=0.4808$, and $\mathrm{R}^{2}=83.9$. MD and $\mathrm{SE}$ values can be interpreted using the antilogs of MD and $S E, 1.012$ and 3.03 , respectively. The former indicates that the average ratio of all the comparison points does not favor overpredicting or underpredicting. the latter indicates that one is $68 \%$ certain that a SPARC-87 estimate will be within a factor of 3 with an experimental measurement. The $R^{2}$ value is $83.9 \%$ of 100 , a perfect correlation of data and experiment.

TABLE 5.1. SPARC-87/ACE DF Comparisons

\begin{tabular}{|c|c|c|c|c|c|}
\hline $\begin{array}{c}\text { Experimental } \\
\text { Data } \\
\end{array}$ & Aerosol & $\begin{array}{c}\text { SPARC } \\
\mathrm{A}\end{array}$ & $\begin{array}{c}\text { SPARC } \\
\mathrm{B}\end{array}$ & $\begin{array}{c}\text { Ratio } \\
\quad \mathrm{A} \\
\end{array}$ & $\begin{array}{c}\text { Ratio } \\
\text { B } \\
\end{array}$ \\
\hline $\begin{array}{r}145.0 \\
47.0 \\
11.0\end{array}$ & $\begin{array}{l}\mathrm{CsOH} \\
\mathrm{CsI} \\
\mathrm{MnO}\end{array}$ & $\begin{array}{r}522.421 \\
196.334 \\
81.295\end{array}$ & $\begin{array}{l}490.444 \\
202.985 \\
113.439\end{array}$ & $\begin{array}{l}3.603 \\
4.177 \\
7.390\end{array}$ & $\begin{array}{r}3.382 \\
4.319 \\
10.313\end{array}$ \\
\hline $\begin{array}{r}840.0 \\
1500.0 \\
260.0\end{array}$ & $\begin{array}{l}\mathrm{CsOH} \\
\mathrm{CsI} \\
\mathrm{MnO}\end{array}$ & $\begin{array}{l}218.971 \\
632.454 \\
141.835\end{array}$ & $\begin{array}{l}452.353 \\
115.701 \\
332.777\end{array}$ & $\begin{array}{l}0.261 \\
0.422 \\
0.546\end{array}$ & $\begin{array}{l}0.539 \\
0.077 \\
1.280\end{array}$ \\
\hline $\begin{array}{r}320.0 \\
220.0 \\
75.0\end{array}$ & $\begin{array}{l}\mathrm{CsOH} \\
\mathrm{CsI} \\
\mathrm{MnO}\end{array}$ & & $\begin{array}{r}1207.978 \\
197.996 \\
60.779\end{array}$ & & $\begin{array}{l}3.775 \\
0.900 \\
0.810\end{array}$ \\
\hline $\begin{array}{r}3000.0 \\
1300.0 \\
180.0\end{array}$ & $\begin{array}{l}\mathrm{CsOH} \\
\mathrm{CsI} \\
\mathrm{MnO}\end{array}$ & $\begin{array}{r}1122.442 \\
882.875 \\
431.878\end{array}$ & $\begin{array}{r}1123.149 \\
637.785 \\
1911.446\end{array}$ & $\begin{array}{l}0.374 \\
0.679 \\
2.399\end{array}$ & $\begin{array}{r}0.374 \\
0.491 \\
10.619\end{array}$ \\
\hline
\end{tabular}

(a) Documented in Allemann and Bamberger, Advanced Containment Experiments, ACE-TR-A13, June 1990. For further information, contact the Electric Power Research Institute. 
TABLE 5.2. SPARC-87/BCL DF Comparisons

$\begin{array}{cccc}\begin{array}{c}\text { Experimental } \\ \text { Data }\end{array} & \begin{array}{c}\text { Vent } \\ \text { Type(a) }\end{array} & \text { SPARC } & \text { Ratio } \\ 1.4 & \text { DC1 } & 1.190 & 0.850 \\ 2.1 & \text { DC2 } & 1.167 & 0.556 \\ 1.4 & \text { DC3 } & 6.793 & 4.852 \\ 6.1 & \text { DC4 } & 2.086 & 0.342 \\ 5.3 & \text { DC5 } & 1.783 & 0.336 \\ 4.7 & \text { DC6 } & 8.359 & 1.779 \\ 2.9 & \text { HV1 } & 1.099 & 0.379 \\ 2.8 & \text { HV2 } & 1.385 & 0.495 \\ 3.8 & \text { HV3 } & 1.266 & 0.333 \\ 1.3 & \text { HV4 } & 2.101 & 1.616 \\ 2.8 & \text { HV5 } & 1.611 & 0.575 \\ 4.0 & \text { HV6 } & 2.419 & 0.605 \\ 3.8 & \text { MH1 } & 2.880 & 0.758 \\ 2.3 & \text { MH2 } & 1.958 & 0.851 \\ 4.6 & \text { MH3 } & 4.115 & 0.895 \\ 3.2 & \text { MH4 } & 3.665 & 1.145 \\ 7.0 & \text { MH5 } & 5.851 & 0.836\end{array}$

(a) $\quad H V=2-f t$ diameter horizontal vent; $D C=2-f t$ diameter downcomer; and $M H=$ multihole spargers $(10,10,4,4$, $1=$ no. of $0.5-i n$. diameter holes in MH1 to MH5, respectively).

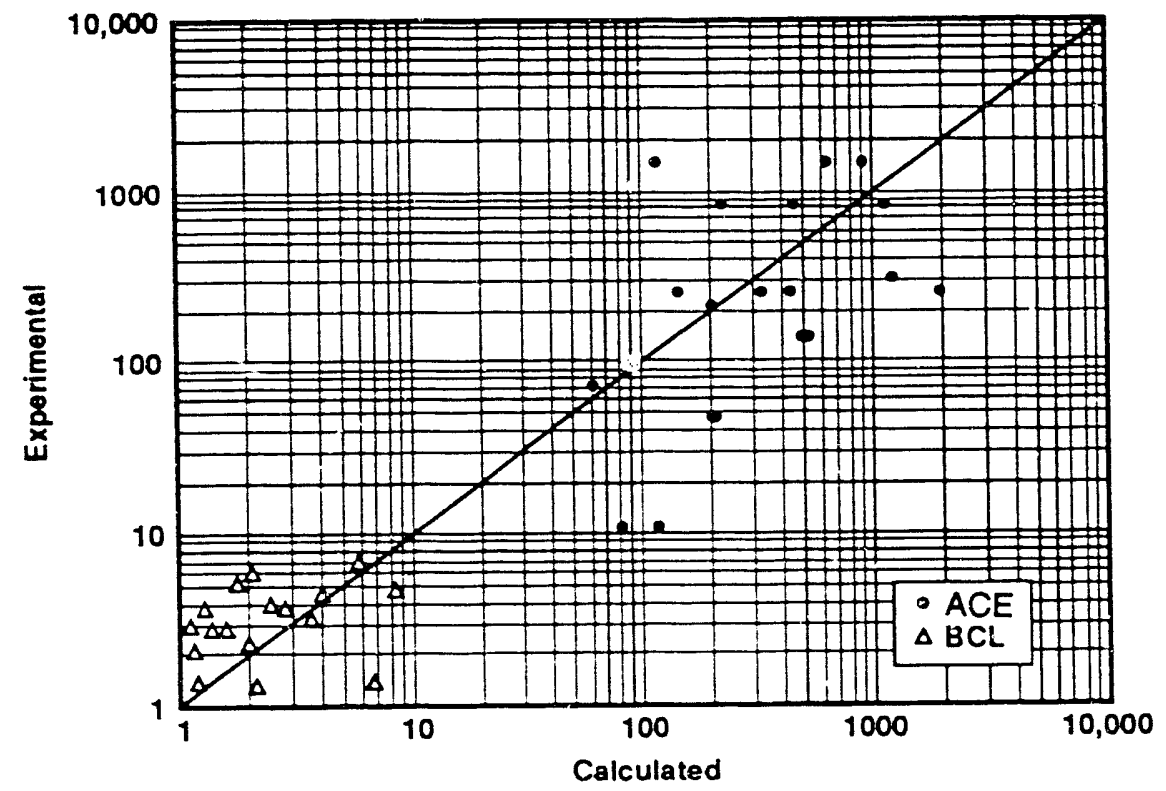

FIGURE 5.1. Log/Log Plot of the Two DF Data Sets of Tables 5.1 and 5.2 


\subsection{SPARC -90 COMPARISON}

The SPARC-90 code was used to calculate DFs in the same way as SPARC-87 for the same two data sets. The comparisons are presented similarly in Tables 5.3 and 5.4 .

Corresponding statistical parameters are $M D=-0.088, S E=0.360$, and $R^{2}=90.8$. The antilogs of MD and SE are 0.817 and 2.29 , respectively. Because these values are not greatly different from those of SPARC-87, one might be concerned about the attainable limit achievable when comparing improved model calculations with experiments. The degree of improvement is certainly limited by the range of uncertainties in the data. A good hint of these uncertainties can be made by comparing the ratio $A$ to ratio $B$ values in Tables 5.1 and 5.3. Here the particle sizes measured produced a high degree of spread in calculated DFs $(A: B)$ for a single DF measured in several experiments. So one concludes that model inadequacies are not the sole reason for differences in model/data comparisons.

Input data files for the SPARC-90/ACE comparisons were compiled in a manner that was probably not the most compatible with the intended use of either SPARC-87 or SPARC-90. The CSI and $\mathrm{CSOH}$ were considered in SPARC-90 input tables as separate aerosol species. Experimentally, the CsI was produced by vapor $\mathrm{I}_{2}$ and solid $\mathrm{CsOH}$ reaction producing a soluble aerosol of mixed species. This mixed aerosol could have been handled by SPARC-90. However, because experimental DFs and particle sizes were supplied in the separated fashion, the separate species comparisons are reported here, even though the mixed aerosols would be preferred and more realistic.

\section{TABLE 5.3. SPARC-30/ACE DF Comparisons}

\section{Experimental} Data

145.0

47.0

11.0

840.0

1500.0

260.0

320.0

220.0

75.0

3000.0

1300.0

180.0

\begin{tabular}{l} 
Aeroso \\
\hline $\mathrm{CsOH}$ \\
$\mathrm{CsI}$ \\
$\mathrm{MnO}$
\end{tabular}

$\mathrm{CsOH}$

CSI

$\mathrm{MnO}$

$\mathrm{CsOH}$

Cs I

$\mathrm{MnO}$

$\mathrm{CsOH}$

CsI

$\mathrm{MnO}$

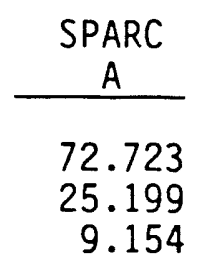
433.741
1057.541
74.353
320.000
220.000
75.000
3436.014
1696.867
264.723

\begin{tabular}{r} 
SPARC \\
$B$ \\
\hline 62.165 \\
26.820 \\
10.094 \\
1009.831 \\
159.924 \\
151.007 \\
2399.003 \\
223.043 \\
49.790 \\
\\
3477.014 \\
1098.989 \\
1033.478
\end{tabular}

1033.478 \begin{tabular}{c}
$\begin{array}{c}\text { Ratio } \\
\mathrm{A}\end{array}$ \\
\hline 0.502 \\
0.536 \\
0.832 \\
0.516 \\
0.705 \\
0.286
\end{tabular}

Ratio

B

0.429

0.571

0.918

1.202

0.107

0.581

7.497

1.014

0.664

1.145

1.159

1.305

1.471

0.845

5.742 
TABLE 5.4. SPARC-90/BCL DF Comparisons

\begin{tabular}{cccc}
$\begin{array}{c}\text { Experin } \\
\text { Dai }\end{array}$ & $\begin{array}{c}\text { Input } \\
\text { File }\end{array}$ & SPARC & Ratio \\
\hline 1.4 & DC1 & 1.251 & 0.894 \\
2.1 & DC2 & 1.221 & 0.581 \\
1.4 & DC3 & 7.891 & 5.636 \\
6.1 & DC4 & 2.580 & 0.423 \\
5.3 & DC5 & 2.041 & 0.385 \\
4.7 & DC6 & 11.363 & 2.418 \\
2.9 & HV1 & 1.148 & 0.396 \\
2.8 & HV2 & 1.593 & 0.569 \\
3.8 & HV3 & 1.384 & 0.364 \\
1.3 & HV4 & 2.110 & 1.623 \\
2.8 & HV5 & 1.616 & 0.577 \\
4.0 & HV6 & 2.429 & 0.607 \\
3.8 & MH1 & 2.659 & 0.700 \\
2.3 & MH2 & 1.546 & 0.672 \\
4.6 & MH3 & 3.463 & 0.753 \\
3.2 & MH4 & 3.728 & 1.165 \\
7.0 & MH5 & 5.971 & 0.853
\end{tabular}

There are assumptions in SPARC-90 that could be replaced with mechanistic models. The first assuription is that the incoming soluble particles are in equilibrium with the incoming gas. The second is that the incoming soluble particles are in equilibrium at $S=0.99$ (if steam is condensing) or $S=0.975$ (if steam is not condensing) after the breakup of the initial globule into the stable bubble swarm. Models needed are those to calculate dynamic heat and mass transfer and the resuiting saturation during initial globule formation and subsequent breakup. 


\subsection{REFERENCES}

Adamson, A. W. 1976. Physical Chemistry of Surfaces. 3rd ed. John Wiley and Sons, New York.

Bird, R. B., W. E. Stewart, and E. N. Lightfoot. 1960. Transport Phenomena. John Wiley and Sons, Inc., New York.

Byers, H. R. 1965. Elements of Cloud Physics. The University of Chicago Press, Chicago, Illinois.

Calderback, P. H., and A. C. Lochiel. 1964. "Mass Transfer Coefficients, Velocities and Shapes of Carbon Dioxide Bubbles in Free Rise Through Distilled Water." Chemical Engineering Science 19:485-503.

Crank, J., 1967. The Mathematics of Diffusion. Oxford University Press, London, England.

Cunane, J.C., et al. 1985. The Scrubbing of Fission Product Aerosols in LWR Water Pools Under Severe Accident Conditions - Experimental Results. EPRI NP-4113-85, Electric Power Research Institute, Palo Alto, California.

Demitrack, T., and F. J. Moody. 1983. "Planetary Ellipsoid Bubble Model for Fission Product Scrubbing." ANS Transactions 45:483-485.

Diffey, H. R. et al. 1965. "Iodine Clean-up in a Steam Suppression System." In International Symposium on Fission Product Release and Transport Under Accident Conditions. CONF-650407, Vol. 2, Oak Ridge National Laboratory, Oak Ridge, Tennessee.

Eggleton, A.E.J. 1967. A Theoretical Examination of Iodine-Water Partition Coefficients. AERE-R 4887, Atomic Energy Research Establishment, Harwell, England.

Fletcher, N. H. 1962. The Physics of Rainclouds. Cambridge University Press, London, England.

Fuchs, N. H. 1964. The Mechanics of Aerosols. The MacMillan Company, New York.

General Electric Company. 1981. 238 Nuclear Island General Electric Standard Safety Analys is Report. (GESSAR II), NRC 22A007, Docket No. STN 50-447, San Jose, California.

Gieske, J. A., P. Cybulski, R. S. Denning, M. R. Kuhlman, K. W. Lee, and H. Chen. 1984. Radionuclide Release Under Specific LWR Accident Conditions, Volume II, BWR Mark I Design. BMI-2104, Battelle Columbus Laboratories, Columbus, Ohio. 
Glew, D. N., and E. A. Moelwyn-Hughes. 1953. "Chemical Statics of the Methyl Halides in Water." In Discussions of the Faraday Society, No. 15, pp. 150161 .

Haberman, W. L., and R. K. Morton. 1953. "Experimental Investigation of the Drag and Shape of Air Bubbles Rising in Various Liquids." David Taylor Model Bas in Report, DTMB No. 802, 55715-102, Carderock, Maryland.

Jokiniemi, J. 1990. The Effect of Airborne Hygroscopic Matter on Aerosol Behavior in Severe Nuclear Power Plant Accidents. Publications 59, Espoo 1990. Technical Research Centre of Finland, Helsinki.

Kataoka, I., and M. Ishii. 1983. Mechanistic Modeling and Correlations for Pool Entrainment Phenomenon. NUREG/CR-3304, ANL-83-37, prepared by Argonne National Laboratory for the U.S. Nuclear Regulatory Commission, Washington, D.C.

Knudsen, J. G. 1970. Properties of Air-Stream Mixture Containing Small Amounts of Iodine. BNWL-1326, Pacific Northwest Laboratory, Richland, Washington.

Moody, F. J., and S. G. Nagy. 1983. "Estimated Effects of Interfacial Vaporization on Fission Product Scrubbing." In Proceedings of ANS-ENS International Meeting on Light Water Reactor Severe Accident Evaluation, pp. 11.5-1 - 11.5-8. American Nuclear Society, LaGrange Park, Illinois.

Owczarski, P. C., and A. K. Postma. 1984. "Suppression Pool Modeling." In Proceedings of the U.S. Nuclear Regulatory Commission Eleventh Water Reactor Safety Research Information Meeting, NUREG/CP-0048, Vol. 3, pp. 469-481. U.S. Nuclear Regulatory Commission, Washington, D.C.

Owczarski, P. C., and A. K. Postma. 1985. "Effects of Water Condensation on Aerosol Particles in PWR Ice Beds and BWR Suppression Pools." In Proceedings of the U.S. Nuclear Regulatory Commission Water Reactor Safety Research Information Meeting, NUREG/CP-0058 Vol. 3, pp. 595-602. U.S. Nuclear Regulatory Commission, Washington, D.C.

Owczarski, P. C., A. K. Postma, and R. I. Schreck. 1985. Technical Bases and User's Manual for the Prototype of SPARC - A Suppression Pool Aerosol Removal Code. NUREG/CR-3317, PNL-4742, prepared by Pacific Northwest Laboratory for the U.S. Nuclear Regulatory Commission, Washington, D.C.

Owczarski, P. C., R. I. Schreck, and W. K. Winegardner. 1985. ICEDF:A Code for Aerosol Particle Capture in Ice Compartments. NUREG/CR-4130, PNL-5379, prepared by Pacific Northwest Laboratory for the U.S. Nuclear Regulatory Commission, Washington, D.C.

Owczarski, P. C., and W. K. Winegardner. 1985. "Validation of SPARC, A Suppression Pool Aerosol Capture Model." Paper IAEA-SM-281/29, presented at IAEA International Symposium on Source Term Evaluation for Accident Conditions, October 28-November 1, 1985, Columbus, Ohio. 
Owczarski, P. C., and W. K. Winegardner. 1986. "Capture of Iodine in Suppression Pool." In Proceedings of the 19th DOE/NRC Nuclear Air Cleaning Conference, NUREC/CP-0086, CONF-860820, Vol. 2. U.S. Nuclear Regulatory Commission, Washington, D.C.

Paul, D. D., L. D. Flanigan, J. C. Cunnane, R. A. Cudnik, R. P. Collier, and R. N. Ohlberg. 1985. "Radionuclide Scrubbing in Water Pools--Gas-Liquid Hydrodynamics." NP-4113-SR, (ANS Topical Meeting, Snowbird, Utah, 1984) Electric Power Research Institute, Palo Alto, California.

Paul, D. D., D. C. Newman, and R. A. Cudnik. 1986. Gas-Liquid Hydrodynamics of Large Vent/Suppression Pool Systems of Nuclear Power Plants During Some Accident Conditions. Electric Power Research Institute, Palo Alto, California.

Rollet, A.-P., R. Cohen-Adad, and C. Ferlin. 1963. "Le Systeme eau-Hydroxyde de Cesium (Water-Cesium Hydroxide System)." Comptes Rendu (Compt. Rend.) $256(56): 5580-5582$.

Zuber, N., and J. A. Findlay. 1965. "Average Volumetric Concentration in Two-Phase Flow Systems." J. Heat Transfer 87:453-468. 
APPENDIX

SPARC-90 LISTING OF INPUT SUBROUTINES 


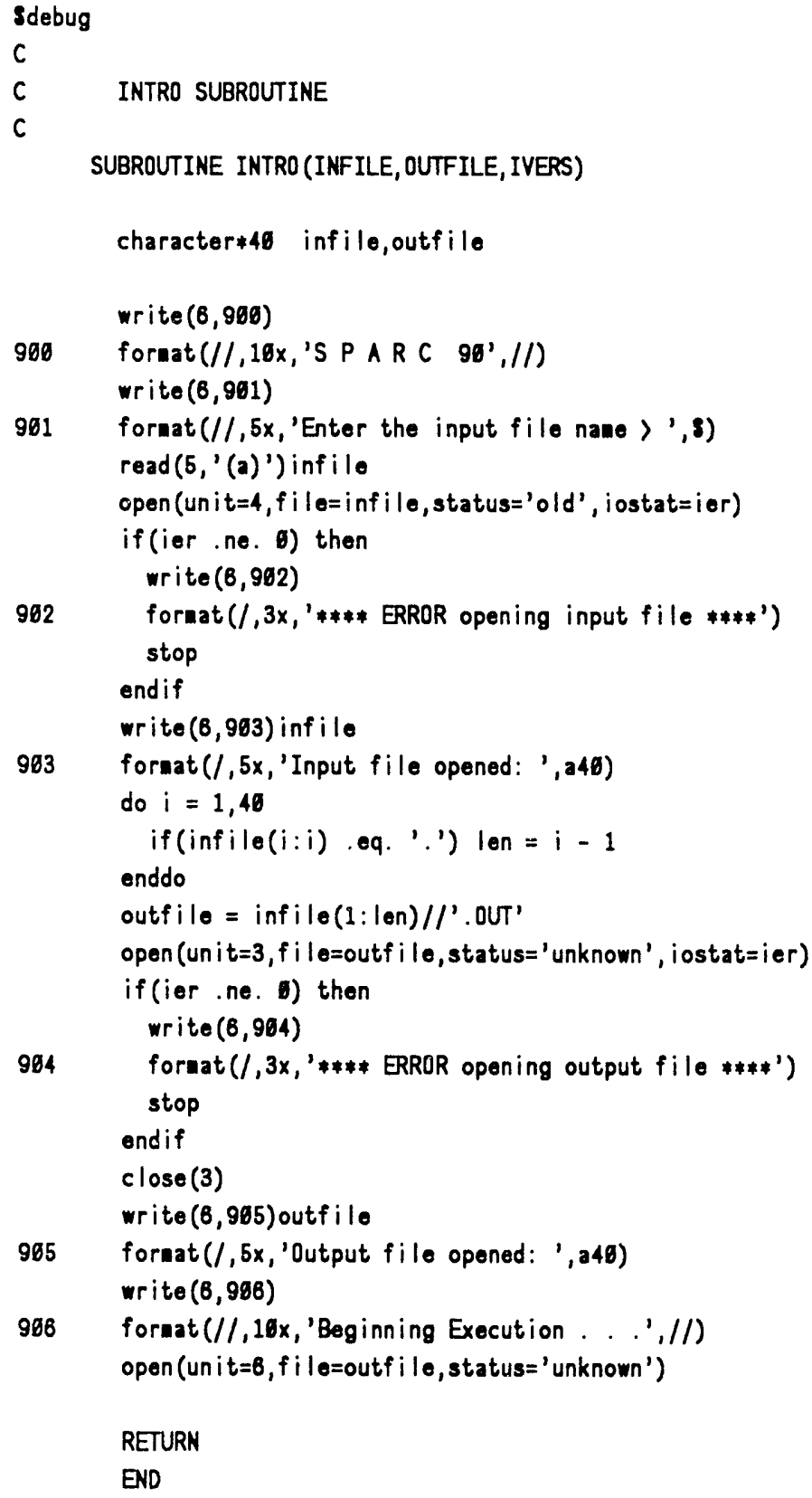


SUBROUTINE INPUT

C

C THIS ROUTINE WILL HANDLE INPUT TO THE MODE

C CALLED FORM THE MAIN CODE.

c

CHARACTER HEADI $* 80$

COMNON / BLKI/PGAS, TGAS, RHOTI, RHOTS, SOLFT, CSFR, RBFR

+ ,ZMSS (20), H2DT, H2ODT, PUDT, DPART (20), DMULT, NBINS, TI2HLF, SUM

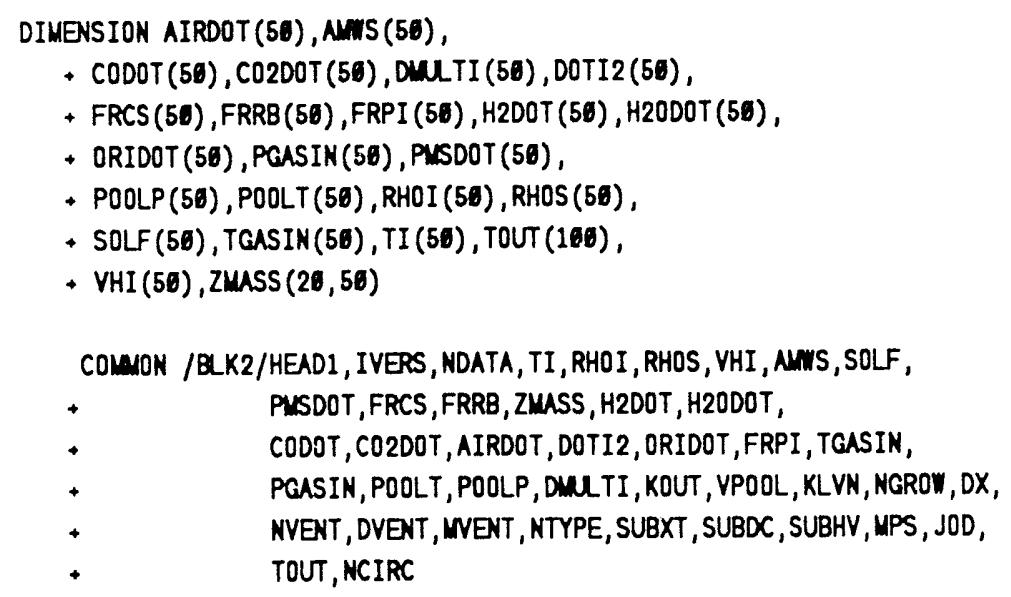









\section{DISTRIBUTION}

No. of

Copies

\section{OFFSITE}

U.S. Nuclear Regulatory Commission

Division of Technical

Information and Document

Control

7920 Norfolk Avenue

Bethesda, MD 20014

5 C. W. Nilsen

Office of Nuclear Regulatory Research

U.S. Nuclear Regulatory Commission

Washington, DC 20555

D. A. COOl

Office of Nuclear Regulatory Research

U.S. Nuclear Regulatory Commission

Washington, DC 20555

R. 0 . Meyer

Office of Nuclear Regulatory Research

U.S. Nuclear Regulatory Commission

Washington, DC 20555

J. A. Mitchell

U.S. Nuclear Regulatory Commission

Washington, DC 20555

K. G. Steyer

Office of Nuclear Regulatory Research

U.S. Nuclear Regulatory Commission

Washington, DC 20555

J. A. Gieseke

Battelle Columbus Laboratories

505 King Avenue

Columbus, $\mathrm{OH} 43201$
No. of

Copies

M. R. Kuhlmann

Battelle Columbus Laboratories 505 King Avenue

Columbus, $\mathrm{OH} \quad 43201$

K. W. Lee

Battelle Columbus Laboratories

505 King Avenue

Columbus, $\mathrm{OH} 43201$

D. D. Paul

Battelle Columbus Laboratories

505 King Avenue

Columbus, $\mathrm{OH} 43201$

H. Ludewig

Bldg. 130

Brookhaven National Laboratory Upton, NY 11973

Wen-Shi Yu

Bldg. 130

Brookhaven National Laboratory Upton, NY 11973

M. Merilo

Electric Power Research Inst. PO Box 10412

Palo Alto, CA 94303

F. J. Rahn

Electric Power Research Inst. PO Box 10412

Palo Alto, CA 94303

R. C. Vogel

Electric Power Research Inst.

PO Box 10412

Palo Alto, CA 94303

D. D. Jones

General Electric Co.

175 Curtner Avenue

San Jose, CA 95125 
NUREG/CR-5765

PNL -7723

No. of

Copies

F. J. Moody

General Electric Co.

175 Curtner Avenue

San Jose, CA 95125

H. E. Townsend

General Electric Co.

175 Curtner Avenue

San Jose, CA 95125

A. K. Postma

Route 1

Box $46 \mathrm{~A}$

Halfway, OR 97834

J. E. Brockmann

Sandia National Laboratories

PO Box 5800

Albuquerque, NM 87185

A. T. Wassel

Science Applications, Inc.

2615 Pacific Coast Highway, Suite 300

Hermosa Beach, CA 90254

Ian B. Wall

81 Irving Avenue

Asherton, CA 94027

\section{International}

A. Alonso

Universidad Politecnica de Madrid Escuela Tecnica Superior de Ingenieros Industriales Laboratorio de Tecnologia Nuclear J. Gutierrez Abascal, 2 28006 Madrid, Spain
No. of

Copies

M. W. Jankowski

International Atomic Energy Agency

Wagramerstrasse 5

PO Box 100

A-1400 Vienna, Austria

S. A. Ramsdale

SRD

Wigshaw Lane

Culcheth

Cheshire WA3 4NE

England

L. Wolf

Battelle-Institut e.V.

Am Römerhof 35

D-6000 Frankfurt am Main 90

Germany

\section{ONSITE}

39 Pacific Northwest Laboratory

R. T. Allemann, K7-15

K. W. Burk, K6-03

E. J. Eschbach, K7-15

J. M. Hales, K6-C4

M. W. Ligotke, P7-54

L. M. Liljegren, K7-15

R. L. Moffitt, K6-46

P. C. Owczarski, K6-08 (20)

J. V. Ramsde 11, K6-03

W. K. Winegardner, K5-i9 (5)

Publishing Coordination

Technical Report Files (5) 
SPARC-90: A Code for Calculating Fission Product

Capture in Suppression Pools

DATE REPORT PUBLISHED

\begin{tabular}{c|r} 
MONTH & VEAR \\
October & 1991
\end{tabular}

4. FIN OR GAANT NUMBER B2444

P. C. Owczarski, K. W. Burk

Technical

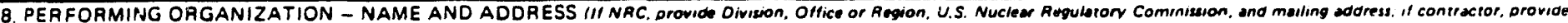
neme and mailing adoress.

Pacific Northwest Laboratory

Richland. WA 99352

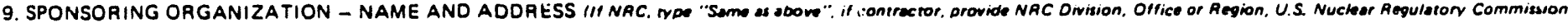
and mailing eddress.)

Division of Regulatory Applications

Office of Nuclear Regulatory Research

U.S. Nuclear Regulatory Commission

Washington, DC 20555

10. SUPPLEMENTARY NOTES

11. ABSTRACT 1200 words of lessi

This report describes the technical bases and use of two updated versions of a computer code initially developed to serve as a tool for calculating aerosol particle retention in boiling water reactor (BWR) pressure suppression pools during severe accidents, SPARC-87 and SPARC-90. The most recent version is SPARC-90. The initial or prototype version (Owczarski, Postma, and Schreck 1985) was improved to include the following: rigorous treatment of local particle deposition velocities on the surface of oblate spherical bubbles, new correlations for hydrodynamic behavior of bubble swarms, models for aerosol particle growth, both mechanistic and empirical models for vent exit region scrubbing, specific models for hydrodynamics of bubble breakup at various vent types, and models for capture of vapor iodine species.

A complete user's guide is provided for SPARC-90 (along with SPARC-87). A code description, code operating instructions, partial code listing, examples of the use of SPARC-90, and summaries of experimental data comparison studies also support the use of SPARC -90 .

12. KEY WORDS/DESCR!PTORS (List words or phreses that will essist resceoreners in loceting ine neport.)

steam suppression pools

aerosol particle retention

bubble swarms

SPARC-90 computer code

\begin{tabular}{|c|}
\hline $\begin{array}{l}\text { 13. AVAILABILITY STATEMENT } \\
\text { Unlimited }\end{array}$ \\
\hline 14. SECUAITY CLASSIFICATION \\
\hline $\begin{array}{l}\text { This Pagel } \\
\text { Unclassified }\end{array}$ \\
\hline $\begin{array}{l}\text { TThis Reoort } \\
\text { Unclass ified }\end{array}$ \\
\hline 15. NUMBEA OF PAGES \\
\hline 16. PRICE \\
\hline
\end{tabular}



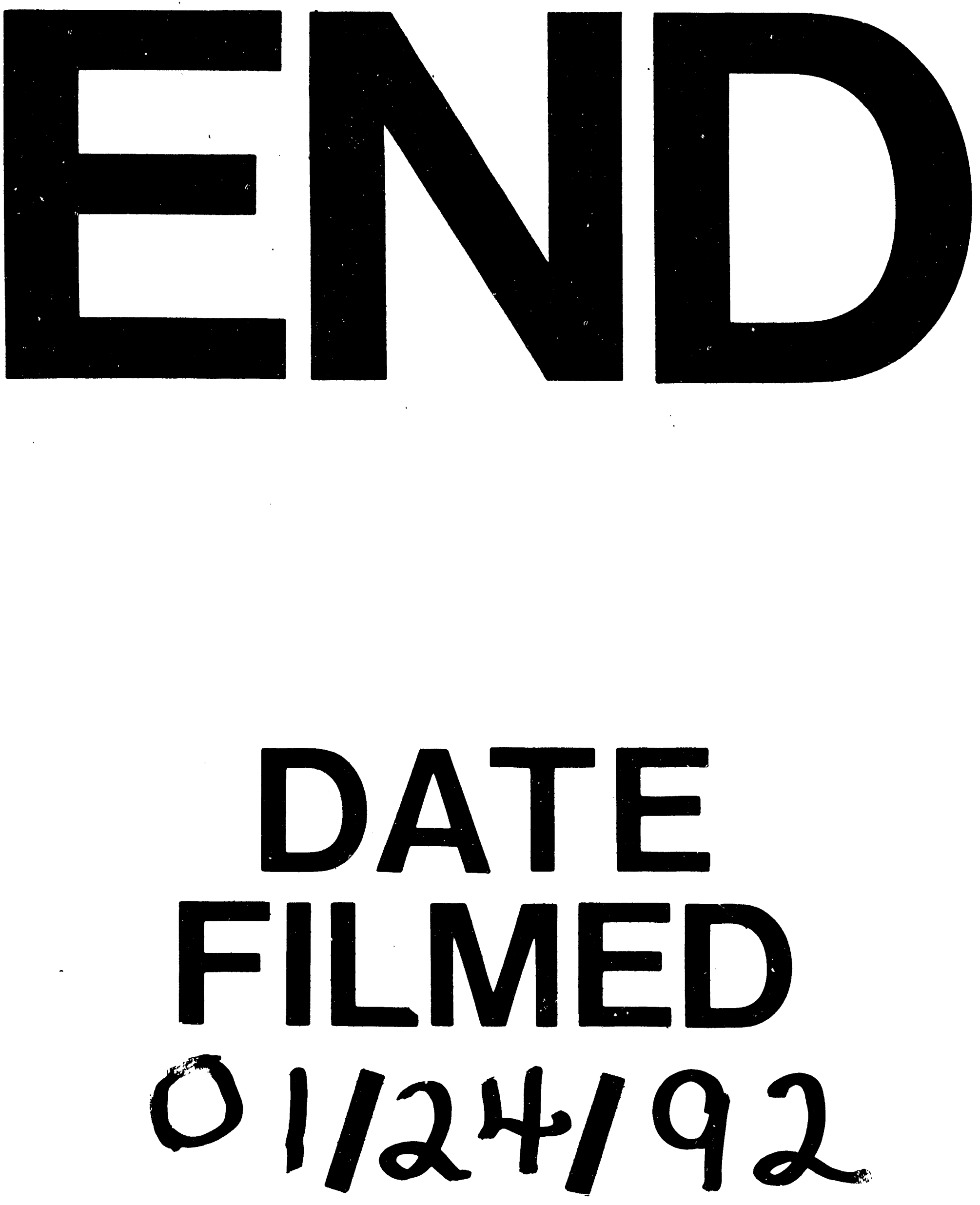